\title{
CLASSIFICATION OF SIMPLE MULTIGERMS OF CURVES IN THE CONTACT SPACE
}

\author{
P. A. KOLGUSHKIN
}

\begin{abstract}
Stably simple singularities of curves (both reducible and irreducible) in the contact complex space are classified up to formal stable contact equivalence.The classification widens the one obtained by V. I. Arnold in 1999 for the simple contact space singularities that are $R L$-equivalent to the singularity $A_{2}$ (a semicubical parabola). The proofs involve the homotopy method and the Darboux-Givental theorem on contact structures.
\end{abstract}

\section{$\S 1$. INTRODUCTION}

A significant number of papers is devoted to classification of simple singularities of curves. Bruce and Gaffney [1] classified the irreducible plane curves. Gibson and Hobbs 22 classified the simple singularities of irreducible curves in the complex 3-space. Arnold [3] classified the (stably) simple singularities of irreducible curves in the linear complex space of an arbitrary dimension. The stably simple singularities of germs of reducible curves (also called multigerms) in the complex space of an arbitrary dimension were classified in [4, 5].

Now suppose that the complex space is equipped with an additional structure, e.g., symplectic or contact. Accordingly, we consider not all local diffeomorphisms of our space, but only those preserving the additional structure.

Suppose that the $2 n$-dimensional complex space is equipped with a symplectic structure and that we are given a germ of a curve equivalent to $\left(t^{2}, t^{2 m+1}\right)$ (the singularity $\left.A_{2 m}\right)$ in the sense of the usual $R L$-equivalence (i.e., we consider all local diffeomorphisms). Arnold investigated this case in [6]. In particular, he showed that this singularity remains simple in the symplectic space. The other stably simple singularities of curves (both irreducible and reducible) were classified up to stable symplectic equivalence in [7.

Assume that the $(2 n+1)$-dimensional complex space is equipped with a contact structure. In this space, we consider a curve singularity $R L$-equivalent to $A_{2}$ (a semicubical parabola). Arnold [8] showed that this singularity does not necessarily remain simple in the contact space. In the same paper, Arnold described the singularity $A_{2}$ in the contact space in the cases where the singularity remains simple. In the present paper, we consider singularities of curves (both irreducible and reducible) in contact spaces and classify simple singularities up to formal stable contact equivalence (which means that the corresponding coordinate changes are determined by formal power series).

Now we give the necessary definitions. A singularity of an irreducible curve at the origin in $\mathbb{C}^{n}$ is a germ of a complex-analytic mapping $f:(\mathbb{C}, 0) \rightarrow\left(\mathbb{C}^{n}, 0\right)$.

2000 Mathematics Subject Classification. Primary 58K40.

Key words and phrases. Contact structure, contact diffeomorphism, multigerm, stably simple singularity.

Partially supported by RFBR (grant no. 01-04-00762) and by grant NSh-1972.2003.1. 
We denote by $L$ the group of coordinate changes in $\left(\mathbb{C}^{n}, 0\right)$, i.e., the group of germs of nondegenerate analytic mappings $\left(\mathbb{C}^{n}, 0\right) \rightarrow\left(\mathbb{C}^{n}, 0\right)$. The group $L$ is called the group of left coordinate changes. Similarly, we denote by $R$ the group of coordinate changes in $(\mathbb{C}, 0)$, i.e., the group of germs of nondegenerate analytic mappings $(\mathbb{C}, 0) \rightarrow(\mathbb{C}, 0)$. The group $R$ is called the group of right coordinate changes.

Definition 1. A multigerm in $\left(\mathbb{C}^{n}, 0\right)$ is a collection $F=\left(f_{1}, \ldots, f_{k}\right)$ of germs of analytic mappings $f_{i}:(\mathbb{C}, 0) \rightarrow\left(\mathbb{C}^{n}, 0\right)$ such that $\operatorname{Im} f_{i} \cap \operatorname{Im} f_{j}=\{0\}$ for $i \neq j$. (The germs $f_{1}, \ldots$, $f_{k}$ are the components of the multigerm.)

Let $G \subset L$ be a subgroup of the group $L$ of left coordinate changes. Then we define $R G=G \times R_{(1)} \times \ldots \times R_{(k)}$, where $R_{(i)}$ is the $i$ th copy of the group $R$ of right coordinate changes. The group $R G$ (of right-left coordinate changes) acts on the space of multigerms by the formula

$$
\left(g, h_{1}, \ldots, h_{k}\right) \cdot\left(f_{1}, \ldots, f_{k}\right)=\left(g \circ f_{1} \circ h_{1}^{-1}, \ldots, g \circ f_{k} \circ h_{k}^{-1}\right) .
$$

Definition 2. A multigerm $F$ is said to be $R G$-simple if a certain neighborhood of $F$ in the space of multigerms (with the usual Whitney topology: the base of the topology consists of the preimages of open sets in the space of $m$-jets for each $m$ ) intersects a finite number of $R G$-orbits. Two multigerms $F$ and $F^{\prime}$ in $\mathbb{C}^{n}$ are said to be $R G$-equivalent if they lie in the same $R G$-orbit.

In what follows, we assume that our space has odd dimension $2 n+1$ and is equipped with a contact structure; i.e., the tangent bundle of the space $\mathbb{C}^{2 n+1}$ is equipped with a distribution of hyperplanes satisfying the condition of "maximal nonintegrability". (Such a distribution is determined by the equation $\alpha=0$, where $\alpha$ is a certain 1-form.) Let $G=$ Cont be the subgroup of $L$ consisting of germs of contact diffeomorphisms, i.e., diffeomorphisms preserving the contact structure. In this case, the $R G$-equivalence will be called contact equivalence. By the Darboux theorem on contact structures, the space $\left(\mathbb{C}^{2 n+1}, 0\right)$ possesses a coordinate system $\left(z, q_{1}, \ldots, q_{n}, p_{1}, \ldots, p_{n}\right)$ in which the 1 -form determining the contact structure is $d z+\sum_{i=1}^{n} p_{i} d q_{i}$.

Now we state Arnold's result on the singularity $A_{2}$ in the contact space, which was proved in [8].

Theorem 1 (V. I. Arnold). Up to contact equivalence, there exist four simple singularities in the contact space $\mathbb{C}^{2 n+1}$ that are $R L$-equivalent to the germ of the semicubical parabola $\left(t^{2}, t^{3}, 0, \ldots, 0\right)$ :

$$
\begin{aligned}
& a^{0}:\left(z=t^{2}, q_{1}=t^{3}, q_{>1}=p=0\right), \\
& b^{1}: \quad\left(z=t^{3}, q_{1}=t^{2}, \quad q_{>1}=p=0\right), \\
& c^{2}: \quad\left(z=t^{4}, \quad q_{1}=t^{2}, \quad p_{1}=t^{3}, \quad q_{>1}=p_{>1}=0\right), \\
& e^{3}: \quad\left(z=t^{4}, q_{1}=t^{2}, q_{2}=t^{3}, p_{1}=t^{5}, q_{>2}=p_{>1}=0\right) \text {. }
\end{aligned}
$$

Remark. The list in [8] contains one more germ:

$$
f^{4}:\left(z=t^{4}, q_{1}=t^{2}, q_{2}=t^{3}, q_{>2}=p=0\right) .
$$

However, this germ turned out to be equivalent to the normal form $e^{3}$ (see Lemma 8).

Arnold [8] also presented a nonsimple (from the contact point of view) germ $R L$-equivalent to $A_{2}$, which is adjoined by all other singularities $R L$-equivalent to $A_{2}$ :

$$
\left(z=a t^{5}, q_{1}=t^{2}, p_{1}=t^{3}, q_{>2}=p_{>2}=0\right) .
$$

We need yet another definition.

Definition 3. A multigerm $F$ in the contact space $\left(\mathbb{C}^{2 n+1}, 0\right)$ is said to be stably simple if $F$ remains simple after the embedding $i:\left(\mathbb{C}^{2 n+1}, 0\right) \hookrightarrow\left(\mathbb{C}^{2 N+1}, 0\right)$ in a contact space 
of higher dimension. Here, the contact structure in the space $\left(\mathbb{C}^{2 N+1}, 0\right)$ is determined by a 1 -form $\alpha$ such that the form $i^{*} \alpha$ determines the initial contact structure on the space $\left(\mathbb{C}^{2 n+1}, 0\right)$.

If two multigerms become contact equivalent after such embeddings, then we say that they are stably equivalent.

We classify the remaining stably simple singularities of multigerms in the contact space up to formal stable contact equivalence (i.e., we use coordinate changes determined by formal power series).

Suppose that we have a coordinate system $\left(z, q_{1}, \ldots, q_{n}, p_{1}, \ldots, p_{n}\right)$ in $\mathbb{C}^{2 n+1}$ in which the form determining the contact structure is standard: $d z+\sum_{i=1}^{n} p_{i} d q_{i}$. We denote by $\left(z \mid q_{1}, \ldots, q_{n} ; p_{1}, \ldots, p_{n}\right)$ the point with coordinates $\left(z, q_{1}, \ldots, q_{n}, p_{1}, \ldots, p_{n}\right)$. If the coordinates $p_{i}$ and $q_{i}$ vanish for all $i>i_{0}$, then sometimes we shall omit the vanishing coordinates in the formulas. Our main result is as follows.

Theorem 2. Up to permutation of components, each stably simple multigerm in the contact space is formally stably equivalent to exactly one multigerm on the following list.

\section{Singularities of irreducible curves.}

\subsection{Regular curves.}

1. $(t \mid 0 ; 0) ; \quad$ 2. $\left(t^{m} \mid t ; 0\right), \quad m>1$.

\subsection{Curves of multiplicity 2 .}
1. $\left(t^{3} \mid t^{2} ; 0\right)$;
2. $\left(t^{2} \mid t^{2 m+1} ; 0\right)$;
3. $\left(t^{4}+t^{2 m+1} \mid t^{2} ; 0\right), \quad m>1$;
4. $\left(t^{4} \mid t^{2} ; t^{2 m+1}\right)$;
5. $\left(t^{4} \mid t^{2}, t^{2 m+1} ; 0,0\right)$.

\subsection{Curves of multiplicity 3 .}
1. $\left(t^{3} \mid t^{4} ; t^{5}\right)$
4. $\left(t^{3} \mid t^{4} ; 0\right)$;
7. $\left(t^{3} \mid t^{5}+t^{7} ; 0\right)$;
2. $\left(t^{3} \mid t^{4}, t^{5} ; 0,0\right)$;
3. $\left(t^{3} \mid t^{4}+t^{5} ; 0\right)$;
5. $\left(t^{3} \mid t^{5} ; t^{7}\right)$;
6. $\left(t^{3} \mid t^{5}, t^{7} ; 0,0\right)$;
10. $\left(t^{3} \mid t^{7}, t^{8} ; t^{11}, 0\right)$;
8. $\left(t^{3} \mid t^{5} ; 0\right)$;
9. $\left(t^{3} \mid t^{7} ; t^{8}\right)$
13. $\left(t^{4} \mid t^{3}, t^{5} ; 0,0\right)$;
11. $\left(t^{3} \mid t^{7}, t^{8} ; 0,0\right)$
12. $\left(t^{4} \mid t^{3} ; t^{5}\right)$
14. $\left(t^{4}+t^{6} \mid t^{3} ; 0\right)$;
15. $\left(t^{4} \mid t^{3} ; 0\right)$.

\section{Singularities of reducible curves.}

\subsection{Multigerms with nonsingular components.}

2.1.1. Case of two components. The first component has the form $(t \mid 0 ; 0)$. We indicate only the second component:
1. $(t \mid t ; 0)$;
2. $\left(t^{m} \mid t ; 0\right), \quad m>1$
3. $\left(t \mid t^{m} ; 0\right)$.

2.1.2. Case of three components. The first two components have the form $((t)$ $0,0 ; 0,0),(t \mid t, 0 ; 0,0))$. We indicate only the third component:
1. $(t \mid 0 ; t)$
2. $(t \mid 0, t ; 0,0)$;
3. $\left(t^{m} \mid 0 ; t\right), m>1$;
4. $\left(t^{m} \mid 0, t ; 0,0\right), m>1$. 
2.2. Multigerms containing a nonsingular and a singular component. Here, the nonsingular component has the form $(t \mid 0,0 ; 0,0)$. We indicate only the singular component:
1. $\left(t^{2} \mid t^{2} ; t^{2 m+1}\right)$
2. $\left(t^{2} \mid t^{2}, t^{2 m+1} ; 0,0\right)$;
4. $\left(t^{3} \mid t^{2} ; t^{3}\right)$;
5. $\left(t^{3} \mid t^{2}, t^{3} ; 0,0\right)$;
3. $\left(t^{2} \mid t^{2}+t^{2 m+1} ; 0\right)$;
7. $\left(t^{3} \mid t^{2} ; 0\right)$;
8. $\left(t^{2} \mid t^{3} ; t^{4}\right)$;
10. $\left(t^{2} \mid t^{3}+t^{4} ; 0\right)$
11. $\left(t^{2} \mid t^{3} ; 0\right)$;
6. $\left(t^{3} \mid t^{2}+t^{3} ; 0\right)$
9. $\left(t^{2} \mid t^{3}, t^{4} ; 0,0\right)$;
13. $\left(t^{2} \mid t^{4}, t^{5} ; t^{7}, 0\right)$;
14. $\left(t^{2} \mid t^{4}, t^{5} ; 0,0\right)$
12. $\left(t^{2} \mid t^{4}, 0 ; t^{5}, 0\right)$;

The rest of the paper is devoted to the proof. In $\S 2$, we state auxiliary assertions. In $\S 3$, we classify singularities of irreducible curves. In $\S \S 4$ and 5 , we consider the case of reducible curves.

\section{§2. Auxiliary assertions}

Suppose that we are given a germ of a contact structure in the space $\mathbb{C}^{2 n+1}$ at the origin. We assume that the contact form is of standard type $d z+\sum_{i=1}^{n} p_{i} d q_{i}$ in the coordinates

$$
\left(z \mid q_{1}, \ldots, q_{n} ; p_{1}, \ldots, p_{n}\right) .
$$

A vector field having the form

$$
\left\{\begin{array}{l}
\dot{z}=K-p K_{p}, \\
\dot{q}=K_{p}, \\
\dot{p}=-K_{q}+p K_{z}
\end{array}\right.
$$

for a certain function $K=K(z, q, p)$ is said to be contact. It is known that, integrating such a vector field, we obtain a one-parameter family of contact diffeomorphisms (see [9]).

We shall need the following result.

Lemma 1. Consider the symplectic form $\sum_{i}^{n} d p_{i} \wedge d q_{i}$ on the space $\mathbb{C}^{2 n}$ with coordinates $(q, p)$. Let

$$
\left\{\begin{array}{l}
Q=Q(q, p) \\
P=P(q, p)
\end{array}\right.
$$

be a symplectomorphism of this space. Then the symplectomorphism lifts to a contact diffeomorphism of the space $\mathbb{C}^{2 n+1}$ with coordinates $(z, q, p)$.

Proof. Since the 1-form $P d Q-p d q$ is closed, the Poincaré lemma implies the existence of a function $S=S(q, p)$ such that $d S=P d Q-p d q$. We easily see that extending the diffeomorphism $(Q, P)$ by putting $Z=z-S(q, p)$ yields a contact diffeomorphism.

We shall apply repeatedly the Darboux-Givental theorem on contact structures [10]. In order to formulate it, we define the restriction of a contact structure to a nonsingular submanifold. We take a 1 -form $\alpha$ determining the contact structure and restrict it to the submanifold. Two forms obtained from each other via multiplying by a nonvanishing function determine one and the same induced structure (see [10]). The class of forms obtained in this way is called the restriction of the contact structure to the nonsingular submanifold. We observe that the structure thus defined is finer than merely the field of tangent subspaces cut out on the submanifold by the hyperplanes of the contact structure.

Theorem 3 (Darboux-Givental). Suppose $N$ is a smooth submanifold of the manifold $M$, and $\gamma_{0}$ and $\gamma_{1}$ are two contact structures on $M$ coinciding on $T N$. Then for each point $x \in N$ there exists a diffeomorphism $U_{0} \rightarrow U_{1}$ between two neighborhoods of $x$ in $M$ that is identical on $N \cap U_{0}$ and maps $\left.\gamma_{0}\right|_{U_{0}}$ to $\left.\gamma_{1}\right|_{U_{1}}$. 


\section{§3. Singularities of IRREDUCible CURVES}

3.1. Singularity of type $A_{2 m}$. We consider a singularity $R L$-equivalent to $\left(t^{2}, t^{2 m+1}\right)$. There are two possibilities: either the tangent space of the germ of a curve at the origin lies in the contact hyperplane, or it does not.

3.1.1. The tangent space at the origin is transversal to the contact hyperplane. First, we assume that the tangent space is transversal to the contact hyperplane. We apply the Darboux-Givental theorem for contact structures. Consider a germ of a two-dimensional submanifold in $\mathbb{C}^{2 n+1}$ containing our curve. Since the tangent space of the curve at the origin is transversal to the contact hyperplane, the distribution of contact hyperplanes cuts out an integrable field of lines on our surface. Using the Darboux-Givental theorem, we reduce the problem to the following one: we are given the line distribution $d z=0$ (horizontal lines) on a two-dimensional (complex) surface with coordinates $(x, z)$. Furthermore, we are given a germ of a curve on the surface that is $R L$-equivalent to $\left(t^{2}, t^{2 m+1}\right)$. We can use only those left coordinate changes that preserve the above distribution of lines, i.e., we can use only the changes of the form

$$
\left\{\begin{array}{l}
\tilde{x}=\tilde{x}(x, z) \\
\tilde{z}=\tilde{z}(z)
\end{array}\right.
$$

Lemma 2. Suppose we are given a curve $R L$-equivalent to $\left(t^{2}, t^{2 m+1}\right)$. If the tangent vector of the curve is transversal to the contact hyperplane, then the curve is contact equivalent to the normal form

$$
\left(t^{2} \mid t^{2 m+1} ; 0\right) .
$$

Proof. It is obvious that in the coordinates $(x, z)$ described above we can bring the curve to the form

$$
\left(a_{1} t^{2}+a_{2} t^{4}+\cdots+a_{m} t^{2 m}+t^{2 m+1}+o\left(t^{2 m+1}\right), t^{2}\right) .
$$

The left change

$$
\left\{\begin{array}{l}
\tilde{x}=\sum_{j=1}^{m} a_{j} z^{j} \\
\tilde{z}=z
\end{array}\right.
$$

brings the curve to the form $\left(t^{2 m+1}+o\left(t^{2 m+1}\right), t^{2}\right)$. However, each function of the form $o\left(t^{2 m+1}\right)$ can be represented as $f(x, z)$. The corresponding left change yields the required normal form.

Now we suppose that the tangent vector of the curve lies in the contact hyperplane, but the tangent space is transversal to the contact hyperplane at the origin. We assume that $m>1$. (The case where $m=1$ was treated by Arnold in 8 .)

Lemma 3. Suppose that we are given a simple curve $R L$-equivalent to $\left(t^{2}, t^{2 m+1}\right)(m>$ 1). We assume that the tangent space of the curve at the origin is transversal to the contact hyperplane, but the tangent vector at the origin lies in the contact hyperplane. Then the curve is contact equivalent to the normal form

$$
\left(t^{4}+t^{2 m+1} \mid t^{2} ; 0\right)
$$

Proof. Obviously, in the coordinates $(x, z)$ described above we can bring the curve to the form

$$
\left(t^{2}+o\left(t^{2}\right), t^{4}+o\left(t^{4}\right)\right)
$$

(If the monomial $t^{4}$ in the coordinate $z$ is absent, then our germ adjoins the nonsimple germ (1.1), whence it follows that our germ itself is nonsimple.) A right-left change brings the germ to the form

$$
\left(t^{2}+t^{2 k+1}+o\left(t^{2 k+1}\right), t^{4}\right)
$$


Such a germ is contact equivalent to $\left(t^{2}+t^{2 k+1}, t^{4}\right)$. Indeed, the monomial $t^{2 r}$ is obtained with the help of $x^{r}$, while $t^{2 k+2 r+1}(r>0)$, with the help of $x^{r-1}\left(x^{2}-z\right)$.

Now we clarify the relationship between $k$ and $m$. For this purpose, we use the right change that brings the coordinate $x$ to the form $t^{2}$. We easily see that this change has the form $t \mapsto t-(1 / 2) t^{2 k}+o\left(t^{2 m}\right)$. After this change, the coordinate $z$ takes the form $t^{4}-2 t^{2 k+3}+o\left(t^{2 k+3}\right)$. Now we easily see that the curve is $R L$-equivalent to $\left(t^{2}, t^{2 k+3}\right)$. It follows that $2 k+3=2 m+1$ and $k=m-1$.

We complete the proof by observing that the normal form indicated in the lemma is contact equivalent to the germ (3.1). (To see this, it suffices to perform a right change.)

3.1.2. The tangent space at the origin lies in the contact hyperplane. Now we consider the second possibility: we assume that the tangent space of the curve at the origin lies in the contact hyperplane. We project the curve to $\mathbb{C}^{2 n}$ with coordinates $(q, p)$. Since the tangent space of the curve at the origin lies in the contact hyperplane (determined by the equation $d z=0$ ), the projection is a germ that is also $R L$-equivalent to $A_{2 m}$. As we already mentioned (see Lemma 1), the space $\mathbb{C}^{2 n}$ has a symplectic structure such that each symplectomorphism of $\mathbb{C}^{2 n}$ lifts to a contact diffeomorphism of $\mathbb{C}^{2 n+1}$.

A symplectic change brings the projection to one of the normal forms listed in [6], after which we lift the corresponding symplectomorphism to a contact diffeomorphism. We observe that the projection lies in a two- or four-dimensional space. Since the singularity (1.1) is not simple, the coordinate $z$ must have the form $t^{4}+o\left(t^{4}\right)$ (possibly, after linear changes in $\mathbb{C}$ and $\mathbb{C}^{2 n+1}$ ).

We shall need several auxiliary assertions, which are similar to Lemmas 1-3 in [8].

Lemma 4. Suppose $A(x, y)$ is a function germ holomorphic at the origin and such that $A\left(t^{2}, t^{2 m+1}\right) \equiv 0$. Then there exists a germ $B(x, y)$ holomorphic at the origin and such that $A(x, y)=B(x, y)\left(y^{2}-x^{2 m+1}\right)$.

Proof. By the well-known division theorem, we have

$$
A(x, y)=a(x)+y b(x)+B(x, y)\left(y^{2}-x^{2 m+1}\right) .
$$

Performing the substitution $x=t^{2}, y=t^{2 m+1}$, we obtain

$$
0=A\left(t^{2}, t^{2 m+1}\right)=a\left(t^{2}\right)+t^{2 m+1} b\left(t^{2}\right),
$$

whence $a(x) \equiv b(x) \equiv 0$.

Lemma 5. For each function $f(t)$ holomorphic at the origin, there exists a function $F(x, y)$ holomorphic at the origin and such that

$$
t^{2 m} f(t)=F\left(t^{2}, t^{2 m+1}\right) .
$$

Proof. Obviously, we have $f(t)=u\left(t^{2}\right)+t v\left(t^{2}\right)$, whence

$$
t^{2 m} f(t)=t^{2 m} u\left(t^{2}\right)+t^{2 m+1} v\left(t^{2}\right) .
$$

It remains to set $F(x, y)=x^{k} u(x)+y v(x)$.

Lemma 6. Suppose $R(x, y)$ and $M(x, y)$ are two function germs holomorphic at the origin, where

$$
R\left(t^{2}, t^{2 m+1}\right)=t^{a}(c+t r(t)), c \neq 0 \text {, and } M\left(t^{2}, t^{2 m+1}\right)=t^{a+2 m} s(t) .
$$

Then there exist two functions $B(x, y)$ and $C(x, y)$ holomorphic at the origin and such that

$$
M(x, y)=B(x, y) R(x, y)+C(x, y)\left(y^{2}-x^{2 m+1}\right) .
$$


Proof. Applying the preceding lemma, we obtain

$$
\frac{t^{a+2 m} s(t)}{t^{a}(c+t r(t))}=t^{2 m} s(t)(c+\operatorname{tr}(t))^{-1}=B\left(t^{2}, t^{2 m+1}\right),
$$

whence

$$
M\left(t^{2}, t^{2 m+1}\right)-R\left(t^{2}, t^{2 m+1}\right) B\left(t^{2}, t^{2 m+1}\right)=0 .
$$

Applying Lemma 1 to the difference on the left-hand side of the latter equality, we obtain the function $C(x, y)$.

We assume that the projection of our curve to $\mathbb{C}^{2 n}$ is a singularity of type $A_{2 m, 0}$ in the notation of Arnold [6. This means that in $\mathbb{C}^{2}$ with coordinates $(p, q)$ the projection is determined by the equation $H(p, q)=0$, where $H(p, q)=p^{2}-q^{2 m+1}$. Then the coordinate $z$ has the form $z=F(p, q)$ (because the tangent plane of the curve lies in the contact hyperplane). After that, we apply the homotopy method as described in the proof of Theorem 3c in [8]. Linearly joining the function $F$ with the function $F_{0}=q^{2}$, we obtain a family of functions $F_{s}$, where $F_{1}=F$ and $F_{s}\left(t^{2}, t^{2 m+1}\right)=t^{4}+s m(t)$, $m(t)=o\left(t^{4}\right)$. We shall look for the contact Hamiltonian $K$ in the form

$$
K=A(p, q)+(z-F(p, q)) B(p, q) .
$$

It was shown in 6 that the functions $A$ and $B$ must satisfy the following homological equations at points of the curve $\{H=0\}$ :

$$
\begin{aligned}
& \text { (a) }\{A, H\}+B\left(\{H, F\}+p H_{p}\right)=0, \\
& \text { (b) }\{F, A\}+A-p A_{p}=m(t), \\
& \text { (c) } A(0)=A_{p}(0)=A_{q}(0)=B(0)=0 .
\end{aligned}
$$

(Here $\{I, J\}=I_{p} J_{q}-I_{q} J_{p}$ is the Poisson bracket.)

In order to solve this system, we consider a quasihomogeneous filtration such that $\operatorname{deg} q=2, \operatorname{deg} p=2 m+1$, and $\operatorname{deg} H=4 m+2$. We observe that the coordinate $z$ has the form

$$
t^{4}+\sum_{j=3}^{m+1} a_{j} t^{2 j}+O\left(t^{2 m+3}\right),
$$

because otherwise either our curve is not equivalent to $A_{2 m}$, or the tangent plane of the curve at the origin is transversal to the contact hyperplane.

First, we find an approximate solution of our system, which means that the pair of functions $A$ and $B$ will satisfy equation (a) precisely, and equation (b) only approximately:

$$
\{F, A\}+A-p A_{p}=t^{a} \bmod t^{a+1}, \quad a \geq 6 .
$$

Thus, we have reduced our equation (b) to the form where $m(t)=O\left(t^{4 m+3}\right)$, which we shall solve precisely.

In order to find an approximate solution, we drop all terms of $F$ that are of higher order than $q^{2}$. Then the term of minimal quasidegree on the left-hand side of (b) is equal to $-2 q A_{p}$. If $a=2 k(k>2)$, then we take $A=p q^{k-1} / 2$, and if $a=2 k+1(k>m)$, then we take $A=-p^{2} q^{k-m} / 4$. Now we must choose the function $B(p, q)$ so that (a) be fulfilled. We can write this equation as follows:

$$
M=B R+C H, \quad M=\{H, A\}, \quad R=\{H, F\}+p H_{p} .
$$

For $F=q^{2}$, we obtain $R=4 t^{2 m+3}+o\left(t^{2 m+3}\right)$, and the leading terms in $F$ do not change the principal part of $R$. Now we estimate the quasidegree of $M$ :

$$
\operatorname{deg} M=\operatorname{deg} H+\operatorname{deg} A-(2 m+3)=\operatorname{deg} A+2 m-1 .
$$


If $a=2 k$, then

$$
\operatorname{deg} M=4 m+2(k-1)>4 m+3 .
$$

On the other hand, if $a=2 k+1$, then we have

$$
\operatorname{deg} M=6 m+1+2(k-m)>4 m+3 .
$$

Applying Lemma 6, we obtain the required functions $B$ and $C$.

Now suppose that $m(t)=O\left(t^{4 m+3}\right)$. Then we take $A=f H$ and obtain an equation for $f$, namely, $-f R=m(t)$. By Lemma 6, this equation is solvable. It remains to set $B=0$, because we have $\{A, H\}=0$ on the curve $\{H=0\}$. Thus, we have proved the following assertion.

Lemma 7. Assume that the tangent plane of a simple germ RL-equivalent to $A_{2 m}$ lies in the contact hyperplane, and the projection of the germ to the symplectic space $\mathbb{C}^{2 n}$ is equivalent to $A_{2 m, 0}$. Then the germ has the contact normal form

$$
\left(t^{4} \mid t^{2} ; t^{2 m+1}\right) \text {. }
$$

Remark. The construction used in the proof was borrowed from [8].

Now we assume that the projection to the symplectic space is equivalent to the germ $A_{2 m, r}$. Since the germ under consideration is simple, we have $z=t^{4}+o\left(t^{4}\right)$, where the degree of the first nonzero monomial of odd degree is at least $2 m+3$.

Lemma 8. A curve of the form

$$
\left(t^{4}+o\left(t^{4}\right) \mid t^{2}, t^{2 m+1} ; t^{2 m+2 r+1}, 0\right), \quad m>1, \quad r \geq 1,
$$

is contact equivalent to a curve of the form

$$
\left(t^{4}+o\left(t^{4}\right) \mid t^{2}, t^{2 m+1} ; 0,0\right) .
$$

(Observe that the projection of the latter curve to the symplectic space is $A_{2 m, 2 m}$.)

Proof. We apply the homotopy method. Consider the family of curves of the form

$$
\left(t^{4}+o\left(t^{4}\right) \mid t^{2}, t^{2 m+1} ; a t^{2 m+2 r+1}+o\left(t^{2 m+2 r+1}\right), O\left(t^{2 r+4}\right)\right), \quad 0 \leq a \leq 1 .
$$

First, we consider the vector field generated by the contact Hamiltonian $K=-q_{1}^{r+1} q_{2}$. On the curve, the field has the form

$$
\left(-t^{2 m+2 r+3} \mid 0,0 ;(r+1) t^{2 m+2 r+1}, t^{2 r+2}\right) .
$$

After that, we consider the vector field generated by the contact Hamiltonian $K=$ $-q_{1}^{r-1} q_{2} z$. On the curve, the field has the form

$$
\left(-t^{2 m+2 r+3}+o\left(t^{2 m+2 r+3}\right) \mid 0,0 ;(r-1) t^{2 m+2 r+1}+o\left(t^{2 m+2 r+1}\right), t^{2 r+2}+O\left(t^{2 r+4}\right)\right) .
$$

The difference of these two vector fields is equal to

$$
\left(o\left(t^{2 m+2 r+3}\right) \mid 0,0 ; 2 t^{2 m+2 r+1}+o\left(t^{2 m+2 r+1}\right), O\left(t^{2 r+4}\right)\right) .
$$

Integrating the difference, we obtain a contact change that brings our curve to the form

$$
\left(t^{4}+o\left(t^{4}\right) \mid t^{2}, t^{2 m+1} ; O\left(t^{2 m+2 r+3}\right), O\left(t^{2 r+4}\right)\right) \text {. }
$$

The proof of Lemma 4 in [6] shows that the projection of such a curve to the symplectic space is symplectically equivalent to $A_{2 m, \geq 2 r+2}$. Obviously, after a finite number of such iterations we obtain the normal form $A_{2 m, 2 m}$.

Finally, we consider a curve the projection of which to the symplectic space is equivalent to $A_{2 m, 2 m}$, and bring this curve to a normal form. We assume that our curve lies in the space $\mathbb{C}^{5}$ with coordinates $(z \mid x, y ; u, v)$. 
Lemma 9. Any curve of the form $\left(t^{4}+o\left(t^{4}\right) \mid t^{2}, t^{2 m+1} ; 0,0\right)$ is contact equivalent to the curve $\left(t^{4} \mid t^{2}, t^{2 m+1} ; 0,0\right)$.

Proof. Our curve is determined by the equations

$$
H(x, y)=x^{2 m+1}-y^{2}=0, \quad u=v=0, \quad \text { and } \quad z=x^{2}+M(x, y) .
$$

(We can write the coordinate $z$ in this form because, as has already been mentioned, the degree of the first monomial of odd degree is at least $2 m+3$.) The proof is obtained by applying the homotopy method. We observe that the contact vector fields generated by the contact Hamiltonians

$$
H_{e}=2 x u+(2 m+1) y v \quad \text { and } \quad H_{h}=2 y u+(2 m+1) x^{2 m} v
$$

do not shift the projection of the curve to the symplectic space $\mathbb{C}^{4}$. Therefore, we shall look for a contact Hamiltonian in the form

$$
K=f(x, y) H_{e}+g(x, y) H_{h} .
$$

We must solve the following system of homological equations on the curve ( $K=A$, $\left.B=0, A=u A_{u}+v A_{v}\right)$ :

$$
\begin{aligned}
& \text { (a) }\{K, H\}=0, \\
& \text { (b) }\{F, K\}=m(t) .
\end{aligned}
$$

The first equation is fulfilled independently of the choice of the functions $f$ and $g$. The second equation takes the form

$$
f\left\{F, H_{e}\right\}+g\left\{F, H_{h}\right\}=m(t) .
$$

As has already been mentioned, $F(x, y)=x^{2}+M(x, y)$. Then we have

$$
\left\{F, H_{e}\right\}=-4 t^{4}+o\left(t^{4}\right) \quad \text { and } \quad\left\{F, H_{h}\right\}=-4 t^{2 m+3}+o\left(t^{2 m+3}\right) .
$$

First, we "kill" all terms that have even degrees not greater than $2 m+2$. If $t^{2 r}(r>2)$ is the monomial of minimal degree, then we put $f=-x^{r-2} / 4$ and $g=0$. After that, the terms of higher degrees may change, but no odd degrees less than $2 m+3$ arise. Then we "kill" the term proportional to $t^{2 m+3}$. For this, we set $f=0$ and $g=$ const. Now, $m(t)$ takes the form $O\left(t^{2 m+4}\right)$, and it suffices to solve the equation

$$
f\left(-4 t^{4}+o\left(t^{4}\right)\right)=a t^{2 m+4}+o\left(t^{2 m+4}\right) .
$$

It remains to apply Lemma 6 .

3.2. Irreducible curves of multiplicity 3. Now assume that our irreducible curve has multiplicity 3 . This means that at least one of the coordinates of the curve has a power series expansion of the form $t^{3}+o\left(t^{3}\right)$.

We describe the three contact changes to be used in what follows. Let $S=S(q)$ be a function. Then the change

$$
\left\{\begin{array}{l}
Z=z+S(q), \\
P=p-\frac{\partial S(q)}{\partial q}, \\
Q=q
\end{array}\right.
$$

is contact. The following change is also contact:

$$
\left\{\begin{array}{l}
Z=z+p q \\
P=-q \\
Q=p
\end{array}\right.
$$


this allows us to permute the $p$ - and $q$-coordinates or some of them. Finally, for the function $S=S(p)$ we consider the change

$$
\left\{\begin{array}{l}
Z=z+\sum_{i} p_{i} \frac{\partial S(p)}{\partial p_{i}}-S(p), \\
P=p \\
Q=q-\frac{\partial S(p)}{\partial p},
\end{array}\right.
$$

which is also contact.

\subsubsection{The tangent vector at the origin is transversal to the contact hyper- plane.}

Lemma 10. Assume that our curve is $R L$-equivalent to $\left(t^{3}, t^{4}, t^{5}\right)$, and the tangent vector of the curve at the origin is transversal to the contact hyperplane. Then the curve is formally contact equivalent (i.e., contact equivalent from the viewpoint of formal changes) to one of the following two normal forms:

1. $\left(t^{3} \mid t^{4} ; t^{5}\right)$

2. $\left(t^{3} \mid t^{4}, t^{5} ; 0,0\right)$.

Proof. In this situation, we bring the 4 -jet to the form $\left(t^{3} \mid t^{4}, 0, \ldots, 0 ; 0,0, \ldots, 0\right)$. Furthermore, the changes (3.2)-(3.4) and, maybe, changes of the form

$$
\left\{\begin{array}{l}
Q_{1}=q_{1}+\alpha q_{2}, \\
Q_{2}=q_{2}, \\
P_{1}=p_{1}, \\
P_{2}=p_{2}-\alpha p_{1}, \\
Z=z
\end{array}\right.
$$

bring the 5-jet to one of the normal forms listed in the lemma.

Among such 5-jets, each one is $R L$-determined; therefore, our curve is contained in a 3-manifold with coordinates $\left(z, p_{1}, q_{1}\right)$ in case 1 , and in a 5-manifold with coordinates $\left(z, q_{1}, q_{2}, p_{1}, p_{2}\right)$ in case 2 . Furthermore, no linear terms are present in the power series expansions of the functions that express the remaining coordinates via those indicated above.

We consider the restrictions of the contact structure to these submanifolds. The restrictions of the corresponding 1-forms at the origin are equal to $d z+p_{1} d q_{1}$ or $d z+$ $p_{1} d q_{1}+p_{2} d q_{2}$, while the exterior differentials of the forms at the origin are equal to $d p_{1} \wedge d q_{1}$ or $d p_{1} \wedge d q_{1}+d p_{2} \wedge d q_{2}$. In its turn, this means that the induced structures are contact. After that, we apply the contact version of the Darboux-Givental theorem.

Thus, we have arrived at the following problem: we have either a curve in $\mathbb{C}^{3}$ with a 5 -jet of the form 1 , or a 5 -jet in $\mathbb{C}^{5}$ of the form 2 . We must show that the jets are determined. We do this in the case of formal coordinate changes, i.e., in the case where the functions describing the transformation of the coordinates are given by formal power series.

1) We start with the first possibility. We assume that the $3 m$-jet $(m>1)$ of our curve has the form

$$
\left(t^{3}+a t^{3 m} \mid t^{4}+b t^{3 m} ; t^{5}+c t^{3 m}\right) .
$$

Integrating the vector field generated by the contact Hamiltonian $K=b \cdot p z^{m}$, we obtain a change that "kills" the monomial $b t^{3 m}$. Similarly, a change corresponding to the vector field generated by $K=-c q \cdot z^{m}$ eliminates $c t^{3 m}$. Finally, a right change "kills" $a t^{3 m}$.

Now we consider the $(3 m+1)$-jet $(m>1)$, which has the form

$$
\left(t^{3}+a t^{3 m+1} \mid t^{4}+b t^{3 m+1} ; t^{5}+c t^{3 m+1}\right) .
$$


Changes generated by the contact Hamiltonians $b \cdot p q z^{m-1}$ and $c \cdot q^{2} z^{m-1}$ eliminate monomials of degree $3 m+1$ in the coordinates $q$ and $p$. A right change "kills" the corresponding monomial in the coordinate $z$.

Finally, we consider the $(3 m+2)$-jet $(m>1)$, which has the form

$$
\left(t^{3}+a t^{3 m+2} \mid t^{4}+b t^{3 m+2} ; t^{5}+c t^{3 m+2}\right) .
$$

In order to eliminate the monomial in the coordinate $q$, we use the contact Hamiltonian $K=b \cdot p q^{2} z^{m-2}$. We "kill" the corresponding monomial in the coordinate $p$ with the help of $K=-c \cdot q^{3} z^{m-2} / 3$, and a right change compensates for the perturbation in the coordinate $z$.

Since at each step we obtain higher and higher powers of the variables, the changes converge to a formal change, i.e., to a power series in $p$ and $q$ and a power series in $t$.

2 ) Now we consider the second possibility: a curve in $\mathbb{C}^{5}$ has a 5 -jet of the form 2 . Once again, we start with the $3 m$-jet. This jet has the form

$$
\left(t^{3}+a t^{3 m} \mid t^{4}+b t^{3 m}, t^{5}+c t^{3 m} ; d t^{3 m}, e t^{3 m}\right) .
$$

A change generated by the contact Hamiltonian $K=-p_{i} z^{m}$ eliminates the perturbation in the coordinate $q_{i}$, and the changes corresponding to the contact Hamiltonians $K=q_{i} z^{m}$ eliminate the perturbations in the coordinates $p_{i}$. A right change eliminates the monomial in the coordinate $z$.

We pass to the $(3 m+1)$-jet:

$$
\left(t^{3}+a t^{3 m+1} \mid t^{4}+b t^{3 m+1}, t^{5}+c t^{3 m+1} ; d t^{3 m+1}, e t^{3 m+1}\right) .
$$

Using the contact Hamiltonian $K=p_{i} z^{m-1} q_{1}$, we eliminate the perturbation in the coordinate $q_{i}$. We eliminate the perturbation in $p_{1}$ with the help of $K=q_{1}^{2} z^{m-1} / 2$, and we eliminate that in $q_{2}$ with the help of $K=q_{1} q_{2} z^{m-1}$.

Finally, we consider the $(3 m+2)$-jet:

$$
\left(t^{3}+a t^{3 m+2} \mid t^{4}+b t^{3 m+2}, t^{5}+c t^{3 m+2} ; d t^{3 m+2}, e t^{3 m+2}\right) .
$$

We eliminate the perturbation in the coordinate $q_{i}$ with the help of the contact Hamiltonian $K=p_{i} z^{m-1} q_{2}$. We eliminate the perturbation in $p_{2}$ with the help of $K=q_{2}^{2} z^{m-1}$, and we eliminate that in $p_{1}$ with the help of $K=q_{1}^{3} z^{m-2}$. The perturbations in the coordinate $z$ are eliminated by right changes.

Now we consider a curve $R L$-equivalent to $\left(t^{3}, t^{4}\right)$.

Lemma 11. Suppose we are given a curve in the contact space. Assume that the curve is $R L$-equivalent to $\left(t^{3}, t^{4}\right)$, and that the tangent vector of the curve at the origin is transversal to the contact hyperplane. Then the curve is contact equivalent to one of the following normal forms:

1. $\left(t^{3} \mid t^{4} ; 0\right)$;

2. $\left(t^{3} \mid t^{4}+t^{5} ; 0\right)$.

Proof. Since the curve is planar, it is contained in a germ of a two-dimensional submanifold in $\mathbb{C}^{2 n+1}$. Since the tangent vector at the origin is transversal to the contact hyperplane, the contact structure cuts out a one-dimensional distribution on the submanifold. The distribution is integrable by the rectification theorem. We assume that $z$ and $q$ are the coordinates on the submanifold, and the distribution is determined by the form $d z$.

We can use changes of the form

$$
\left\{\begin{array}{l}
\tilde{z}=\tilde{z}(z) \\
\tilde{q}=\tilde{q}(z, q) .
\end{array}\right.
$$


First, we bring our curve with coordinates $(z, q)$ to the form $\left(t^{3}, t^{4}+a t^{5}+o\left(t^{5}\right)\right)$. Since each function of the form $o\left(t^{5}\right)$ can be written as $f(z, q)$ for a certain holomorphic function $f$, the curve is 5 -determined. For $a \neq 0$, we obtain the normal form 2 , and for $a=0$ we obtain the normal form 1.

Thus, we have settled the case of curves with the invariant pair $(3,4)$. We pass to curves with the invariant pair $(3,5)$. Up to $R L$-equivalence, there are only two such curves: $\left(t^{3}, t^{5}, t^{7}\right)$ and $\left(t^{3}, t^{5}\right)$.

Lemma 12. Suppose we are given a curve in the contact space. Assume that the curve is $R L$-equivalent to $\left(t^{3}, t^{5}, t^{7}\right)$, and that the tangent vector of the curve at the origin is transversal to the contact hyperplane. Then the curve is (formally) contact equivalent to one of the following two normal forms:

1. $\left(t^{3} \mid t^{5} ; t^{7}\right)$

2. $\left(t^{3} \mid t^{5}, t^{7} ; 0,0\right)$.

Proof. The arguments are much the same as those in the proof of Lemma 10.

Lemma 13. Suppose we are given a curve in the contact space. Assume that the curve is $R L$-equivalent to $\left(t^{3}, t^{5}\right)$, and that the tangent vector of the curve at the origin is transversal to the contact hyperplane. Then the curve is contact equivalent to one of the following two normal forms:

1. $\left(t^{3} \mid t^{5} ; 0\right)$;

2. $\left(t^{3} \mid t^{5}+t^{7} ; 0\right)$.

Proof. We may argue much as in the proof of Lemma 11.

Now we consider curves with the invariant pair $(3,7)$. From the viewpoint of $R L$-equivalence, the curve $\left(t^{3}, t^{7}, t^{8}\right)$ is the most general of them.

Lemma 14. Suppose we are given a curve in the space $\mathbb{C}^{2 n+1}$ with a contact structure. Assume that the curve is $R L$-equivalent to $\left(t^{3}, t^{7}, t^{8}\right)$, and that the tangent vector of the curve at the origin is transversal to the contact hyperplane. Then the curve is contact equivalent to one of the following three normal forms:

1. $\left(t^{3} \mid t^{7} ; t^{8}\right)$

2. $\left(t^{3} \mid t^{7}, t^{8} ; t^{11}, 0\right)$

3. $\left(t^{3} \mid t^{7}, t^{8} ; 0,0\right)$.

Proof. We observe that we can bring the 8-jet to the form 1 or to the form 3 . Furthermore, applying the argument used in the proof of Lemma 10, we can assume that our curve lies in $\mathbb{C}^{3}$ or in $\mathbb{C}^{5}$.

1) We start with the first possibility: the 8-jet of our curve has the form 1 . Then we eliminate the perturbations in the $3 m$-jet in the same way as in the proof of Lemma 10. The $(3 m+1)$-jet can also be treated in the same way as in the proof of Lemma 10, we only need to reduce by 1 the exponent of the variable $z$ in the formulas for the contact Hamiltonians $K$.

The situation with $(3 m+2)$-jets is somewhat more complicated. The arguments used in the proof of Lemma 10 are valid for $m \geq 4$. For $m=3$, i.e., for the 11-jet, an additional argument is required. So, we assume that the 11 -jet has the form

$$
\left(t^{3}+a t^{11} \mid t^{7}+b t^{11} ; t^{8}+c t^{11}\right) .
$$

A change generated by the contact Hamiltonian $K=b \cdot z p^{2} / 2$ eliminates the perturbation in the coordinate $q$. In order to eliminate the perturbation in the coordinate $p$, we use the 
following three vector fields (we indicate their action on the 11-jet under consideration): one vector field in the tangent space of the $R$-orbit (it corresponds to the vector $t^{4}$ in the Lie algebra of the group $R$ and yields the jet $\left.\left(3 t^{6} \mid 7 t^{10} ; 8 t^{11}\right)\right)$ and two vector fields in the tangent space of the Cont-orbit, which yield $\left(0 \mid t^{10} ;-t^{11}\right)(K=p q z)$ and $\left(t^{6} \mid 0 ; 2 t^{11}\right)$ $\left(K=z^{2}\right)$. The vector $\left(0 \mid 0 ; t^{11}\right)$ is a linear combination of these vectors, which proves the existence of a change "killing" the monomial $c t^{11}$. As usual, the monomial $a t^{11}$ is eliminated by a right change.

2) Now we analyze the second possibility: the 8-jet of our curve has the form 3 . Appropriately adjusting the arguments used in the proof of Lemma 10, we eliminate the monomials of degrees 9 and 10 .

We consider the 11-jet

$$
\left(t^{3}+a t^{11} \mid t^{7}+b t^{11}, t^{8}+c t^{11} ; d t^{11}, e t^{11}\right) .
$$

Using the contact Hamiltonians $K=p_{i} q_{2} z$, we eliminate the perturbations in the coordinates $q_{i}$, and we eliminate the monomial $e t^{11}$ with the help of $K=-e \cdot q_{2}^{2} z / 2$. After an appropriate right change, we may assume that $a=0$. If $d=0$, we obtain the normal form 3 , while if $d \neq 0$, we obtain the normal form 2 .

Observe that the monomial $d t^{11}$ cannot actually be "killed". Indeed, otherwise the tangent space of the orbit containing the 11-jet $\left(t^{3} \mid t^{7}, t^{8} ; 0,0\right)$ would contain the vector $\left(0 \mid 0,0 ; t^{11}, 0\right)$. However, the monomial $t^{11}$ in the coordinate $p_{1}$ can be obtained only with the help of the contact Hamiltonian $K=-q_{1} q_{2} z$. The Hamiltonian determines the vector $\left(0 \mid 0,0 ; t^{11}, t^{10}\right)$, but the monomial $t^{10}$ in the coordinate $p_{2}$ is not eliminated by anything. Therefore, the 11-jets 2 and 3 are not equivalent. The higher jets are treated in the same way as in the proof of Lemma 10.

Now we assume that the 8 -jet has the form $\left(t^{3} \mid t^{7}+a t^{8}, 0, \ldots, 0 ; 0,0, \ldots, 0\right)$. The perturbations in the 9- and 10-jets are eliminated, and in the most general situation, we bring the 11-jet to the form $\left(t^{3} \mid t^{7}+a t^{8} ; t^{11}\right)$, where the monomial $t^{11}$ cannot be eliminated.

We assert that the parameter $a$ is a modulus. Indeed, we must control the behavior of four parameters. Let us find the dimension of the group with the help of which we can change the parameters. Clearly, the left and right changes constituting this group must be linear. Furthermore, if we multiply the coordinates $q$ and $p$ by $\alpha$ and $\beta$, respectively, then a transformation preserves the contact structure only if the coordinate $z$ is multiplied by the product $\alpha \beta$. Therefore, the dimension of the group is equal to $1+2=3$. (The dimension of the group of right changes is equal to 1.) It follows that the parameter $a$ is a modulus. Thus, we have proved the following assertion.

Lemma 15. No curve with the 8-jet $\left(t^{3} \mid t^{7}+a t^{8}, 0, \ldots, 0 ; 0,0, \ldots, 0\right)$ is simple.

It is clear that, in the case where the tangent vector at the origin is transversal to the contact hyperplane, the remaining curves of multiplicity 3 adjoin this singularity and, consequently, are not simple.

3.2.2. The tangent vector at the origin lies in the contact hyperplane. In this situation, we can bring the 4 -jet of the curve to the form $\left(t^{4} \mid t^{3}, 0, \ldots, 0 ; 0,0, \ldots, 0\right)$, since otherwise the curve would have adjoined the singularity (1.1) and thus would not be simple. Up to $R L$-equivalence, there exist only two such curves: $\left(t^{3}, t^{4}, t^{5}\right)$ and $\left(t^{3}, t^{4}\right)$. We handle these two cases in Lemmas 16 and 17, respectively.

Lemma 16. Suppose we are given a simple curve in the space $\mathbb{C}^{2 n+1}$ with a contact structure. Assume that the curve is $R L$-equivalent to $\left(t^{3}, t^{4}, t^{5}\right)$, and that the tangent vector of the curve at the origin lies in the contact hyperplane. Then the curve is equivalent to 
one of the following two normal forms:

1. $\left(t^{4} \mid t^{3} ; t^{5}\right)$;

2. $\left(t^{4} \mid t^{3}, t^{5} ; 0,0\right)$.

Proof. We easily bring the 5-jet of the curve to one of the two forms indicated above. Among such 5-jets, each one is 5-determined (from the viewpoint of $R L$-equivalence); therefore, by the Darboux-Givental theorem, we may assume that our curve lies in a three- or five-dimensional space.

1 ) We start with the first possibility. The $3 m$-jet of the curve $(m \geq 2)$ has the form

$$
\left(t^{4}+a t^{3 m} \mid t^{3}+b t^{3 m} ; t^{5}+c t^{3 m}\right) .
$$

A right change brings the $3 m$-jet to the form

$$
\left(t^{4} \mid t^{3}+a t^{3 m-1}+b t^{3 m} ; t^{5}+c t^{3 m}\right) .
$$

(Here, the coefficients $a, b$, and $c$ may have changed; we preserved this notation only for convenience.) A change generated by the contact Hamiltonian $K=a \cdot p^{2} q^{m-2} / 2$ eliminates the coefficient $a$. It is possible that the coefficients $b$ and $c$ have changed somehow.

After that, a right change makes $b=0$. Finally, the contact Hamiltonian $K=-c \cdot \frac{q^{m+1}}{m+1}$ allows us to "kill" the monomial $c t^{3 m}$.

We pass to the $(3 m+1)$-jet:

$$
\left(t^{4}+a t^{3 m+1} \mid t^{3}+b t^{3 m+1} ; t^{5}+c t^{3 m+1}\right) .
$$

As before, a right change brings this jet to the form

$$
\left(t^{4} \mid t^{3}+a t^{3 m}+b t^{3 m+1} ; t^{5}+c t^{3 m+1}\right) .
$$

The contact Hamiltonian $K=a \cdot p q^{m}$ allows us to "kill" $a$. After that, a right change eliminates the monomial $b t^{3 m+1}$, while a change generated by the contact Hamiltonian $K=-c \cdot z q^{m} / m$ eliminates $c t^{3 m+1}$.

Finally, we consider the $(3 m+2)$-jet:

$$
\left(t^{4}+a t^{3 m+2} \mid t^{3}+b t^{3 m+2} ; t^{5}+c t^{3 m+2}\right) .
$$

Once again, a right change brings this $(3 m+2)$-jet to the form

$$
\left(t^{4} \mid t^{3}+a t^{3 m+1}+b t^{3 m+2} ; t^{5}+c t^{3 m+2}\right) .
$$

We eliminate the monomial $a t^{3 m+1}$ with the help of the contact Hamiltonian $K=a$. $p z q^{m-1}$. After that, a right change eliminates $b t^{3 m+2}$, and we eliminate $c t^{3 m+2}$ with the help of $K=-c \cdot \frac{z^{2} q^{m-1}}{m-1}$.

2) Now we consider the second possibility: we assume that the 5-jet of the curve has the form 2 . We consider the $3 m$-jet $(m \geq 2)$ :

$$
\left(t^{4}+a t^{3 m} \mid t^{3}+b t^{3 m}, t^{5}+c t^{3 m} ; d t^{3 m}, e t^{3 m}\right) .
$$

A right change yields the $3 m$-jet

$$
\left(t^{4} \mid t^{3}+a t^{3 m-1}+b t^{3 m}, t^{5}+c t^{3 m} ; d t^{3 m}, e t^{3 m}\right) .
$$

A change generated by the contact Hamiltonian $K=a \cdot p_{1} q_{1}^{m-2} q_{2}$ eliminates the monomial $a t^{3 m-1}$. A right change eliminates $b t^{3 m}$. In order to "kill" $c t^{3 m}$, we take $K=c \cdot q_{1}^{m} p_{2}$. We eliminate $d t^{3 m}$ with the help of $K=-d \cdot \frac{q_{1}^{m+1}}{m+1}$. Finally, in order to "kill" $e t^{3 m}$, we take $K=-e \cdot q_{1}^{m} q_{2}$.

Now we consider the $(3 m+1)$-jet:

$$
\left(t^{4}+a t^{3 m+1} \mid t^{3}+b t^{3 m+1}, t^{5}+c t^{3 m+1} ; d t^{3 m+1}, e t^{3 m+1}\right) .
$$


A right change yields the $(3 m+1)$-jet

$$
\left(t^{4} \mid t^{3}+a t^{3 m}+b t^{3 m+1}, t^{5}+c t^{3 m+1} ; d t^{3 m+1}, e t^{3 m+1}\right) .
$$

In order to eliminate $a t^{3 m}$, we take $K=a \cdot q_{1}^{m} p_{1}$. A right change "kills" $b t^{3 m+1}$. In order to eliminate $c t^{3 m+1}$, we take $K=c \cdot z q_{1}^{m-1} p_{2}$. A change generated by the contact Hamiltonian $K=-d \cdot z q_{1}^{m}$ eliminates the monomial $d t^{3 m+1}$. Finally, in order to eliminate $e t^{3 m+1}$, we take $K=-e \cdot z q_{1}^{m-1} q_{2}$.

We pass to the $(3 m+2)$-jet:

$$
\left(t^{4}+a t^{3 m+2} \mid t^{3}+b t^{3 m+2}, t^{5}+c t^{3 m+2} ; d t^{3 m+2}, e t^{3 m+2}\right) .
$$

A right change yields a $(3 m+2)$-jet of the form

$$
\left(t^{4} \mid t^{3}+a t^{3 m+1}+b t^{3 m+2}, t^{5}+c t^{3 m+2} ; d t^{3 m+2}, e t^{3 m+2}\right) .
$$

In order to eliminate $a t^{3 m+1}$, we take $K=a \cdot p_{1} z q_{1}^{m-1}$. A right change "kills" $b t^{3 m+2}$. In order to eliminate the monomial $c t^{3 m+2}$, we take $K=c \cdot q_{2} q_{1}^{m-1} p_{2}$. In order to make $e=0$, we take $K=-e \cdot q_{2}^{2} q_{1}^{m-1} / 2$. Finally, in order to eliminate $d t^{3 m+2}$, we take $K=-d \cdot \frac{z^{2} q_{1}^{m-1}}{m-1}$.

Lemma 17. Suppose we are given a simple curve in the contact space $\mathbb{C}^{2 n+1}$. Assume that the curve is $R L$-equivalent to $\left(t^{3}, t^{4}\right)$, and that the tangent vector of the curve at the origin lies in the contact hyperplane. Then the curve is contact equivalent to one of the following two normal forms:

1. $\left(t^{4}+t^{6} \mid t^{3} ; 0\right)$

2. $\left(t^{4} \mid t^{3} ; 0\right)$.

Proof. Since the curve is planar, the germ of the curve is contained in a germ of a twodimensional submanifold. As has already been mentioned, since the curve is simple, the tangent space of the curve is transversal to the contact hyperplane. Therefore, the contact structure cuts out an integrable one-dimensional distribution on our surface. Let $(z, x)$ be the coordinates on the surface in which the distribution is determined by the form $d z=0$.

We can use left changes of the form

$$
\left\{\begin{array}{l}
\tilde{z}=\tilde{z}(z) \\
\tilde{x}=\tilde{x}(x, z)
\end{array}\right.
$$

We observe that the 4-jet of our curve can be brought to the form 2 . Now, a right change and a linear left change bring the curve to the form $\left(z=t^{4}, x=t^{3}+O\left(t^{5}\right)\right)$. Clearly, each function of the form $O\left(t^{6}\right)$ can be written as $f(x, z)$. Therefore, the change

$$
\left\{\begin{array}{l}
\tilde{z}=z \\
\tilde{x}=x-f(x, z)
\end{array}\right.
$$

brings our curve to the form $\left(z=t^{4}, x=t^{3}+a t^{5}\right)$. If $a=0$, then we obtain the normal form 2. Now, if $a \neq 0$, then we obtain the normal form 1 . Indeed, take the curve indicated in the normal form 1 , and perform a right change after which the coordinate $z$ takes the form $t^{4}$. Then the coordinate $x$ takes the form $t^{3}+a t^{5}+o\left(t^{5}\right)$, where $a \neq 0$.

3.3. Irreducible curves of multiplicity 4. Here, we show that no curves of multiplicity 4 are simple from the contact point of view. We observe that, in the generic case, the 6 -jet can be brought to the form $\left(t^{4} \mid t^{5}, 0, \ldots, 0 ; t^{6}, 0, \ldots, 0\right)$. We pass to the 7 -jet. In the generic case, we easily bring this jet to the form $\left(t^{4} \mid t^{5}+a t^{7}, t^{7} ; t^{6}+b t^{7}, 0\right)$.

Lemma 18. The 7-jet $\left(t^{4} \mid t^{5}+a t^{7}, t^{7} ; t^{6}+b t^{7}, 0\right)$ is not simple, the parameters $a$ and $b$ being moduli. 
Proof. A direct check shows that the tangent space of the orbit containing such a 7 -jet does not contain the vectors $\left(0 \mid t^{7}, 0 ; 0,0\right)$ and $\left(0 \mid 0,0 ; t^{7}, 0\right)$.

\section{§4. Singularities of multigerms with Regular COMPonents}

In this section and in the next one, we consider simple singularities of germs of reducible curves in the contact space.

4.1. Two-component multigerms. We assume that our multigerm consists of two regular components. Up to $R L$-equivalence, they are $((t, 0),(0, t))$ and $\left((t, 0),\left(t, t^{m}\right)\right)$, $m>1$.

We start with the first possibility. We also assume that one of the components (e.g., the first one) is transversal to the contact hyperplane at the origin. This means that the component can be brought to the form $(t \mid 0, \ldots, 0 ; 0, \ldots, 0)$.

Lemma 19. Let $F$ be a multigerm in the contact space. Assume that $F$ is $R L$-equivalent to $((t, 0),(0, t))$, and that the first component of $F$ is transversal to the contact hyperplane. Then the multigerm is contact equivalent to one of the following normal forms (the first component has the form $(t \mid 0 ; 0)$, and so in this list we indicate only the second component):

1. $(t \mid t ; 0)$

2. $\left(t^{m} \mid t ; 0\right), m>1$.

Proof. We assume that the first component already has the standard form. There exists a germ of a two-dimensional surface containing our multigerm. Since the first component is transversal to the contact hyperplane, the contact structure cuts out a one-dimensional distribution on the surface. Let $(z, q)$ be the coordinates on the surface, the distribution being determined by the form $d z=0$.

1) First, we assume that the second component is also transversal to the contact hyperplane. Then, obviously, our multigerm can be brought to the form $((t, 0),(t, t+$ $\left.O\left(t^{2}\right)\right)$ in the coordinates $(z, q)$. It is clear that a function of the form $O\left(t^{2}\right)$ can be written as $z \cdot f(q)$, where $f(0)=0$. The change $(\tilde{z}=z, \tilde{q}=q-z \cdot f(q))$ yields the normal form 1 .

2) Now we assume that the second component touches the contact hyperplane. Then we bring our multigerm to the form $\left((t, 0),\left(t^{m}, t+O\left(t^{2}\right)\right)\right)$ in the coordinates $(z, q)$. A function of the form $O\left(t^{2}\right)$ can be written as $f(q)$. The change $(\tilde{z}=z, \tilde{q}=q-f(q))$ brings our multigerm to the normal form 2 . We observe that the coordinate $z$ must contain the monomial $t^{m}$. Otherwise, the multigerm will adjoin a multigerm with normal form 2 for each $m$ and, thus, will be nonsimple.

Lemma 20. Let $F$ be a multigerm in the contact space. Assume that $F$ is RL-equivalent to $\left((t, 0),\left(t, t^{m}\right)\right)$, and that the tangent vector of the components (since the components touch each other, their tangent vectors at the origin coincide) is transversal to the contact hyperplane. Then the multigerm is contact equivalent to the normal form

$$
\left((t \mid 0 ; 0),\left(t \mid t^{m} ; 0\right)\right) .
$$

Proof. As before, our multigerm is contained in a two-dimensional submanifold with coordinates $(z, q)$, on which the contact structure cuts out the distribution $d z=0$. In these coordinates, we bring the multigerm to the form $\left((t, 0),\left(t, t^{m}+o\left(t^{m}\right)\right)\right.$. It is clear that any function of the form $o\left(t^{m}\right)$ can be written as $q \cdot f(z)$, where $f(0)=0$. Then the change $(\tilde{z}=z, \tilde{q}=q-q \cdot f(z))$ brings the multigerm to the required normal form. 
Now suppose that both components of a multigerm $R L$-equivalent to $((t, 0),(0, t))$ touch the contact hyperplane. We project the multigerm to the space $\{z=0\}$ along the $z$-axis. We observe that in this situation the projections of both components are regular curves. We can assume that the space $\{z=0\}$ is symplectic with Darboux coordinates $(q ; p)$. In the generic case, a multigerm consisting of two regular components in the symplectic space can be brought to the form $\left(\left(t_{1} ; 0\right),\left(0 ; t_{2}\right)\right)$. As we already know, each symplectomorphism of the space lifts to a contact diffeomorphism of the ambient space. Therefore, in the generic case we can bring the 2 -jet to the form $\left(\left(t_{1}^{2} \mid t_{1} ; 0\right),\left(\alpha t_{2}^{2} \mid 0 ; t_{2}\right)\right)$.

Lemma 21. In the family of 2-jets

$$
\left(\left(t_{1}^{2} \mid t_{1} ; 0\right),\left(\alpha t_{2}^{2} \mid 0 ; t_{2}\right)\right)
$$

in the contact space, the parameter $\alpha$ is a modulus, i.e., such a 2-jet is not simple from the contact point of view.

Proof. Should 2-jets be equivalent for distinct values of the parameter $\alpha$, the equivalence would clearly be linear. Now, direct computations easily show that the parameter $\alpha$ is a modulus.

Thus, we have completed consideration of simple multigerms consisting of two regular components.

\subsection{Three-component multigerms.}

4.2.1. First, we assume that our multigerm is $R L$-equivalent to $((t, 0,0),(0, t, 0),(0,0, t))$. Lemma 21 implies that if the multigerm is simple, then at least two of its components are transversal to the contact hyperplane. Therefore, we can assume that the first two components have the normal form 1 of Lemma 19.

Lemma 22. Let $F$ be a multigerm in the contact space. Assume that $F$ is $R L$-equivalent to $((t, 0,0),(0, t, 0),(0,0, t))$, and that the first two components are transversal to the contact hyperplane at the origin. Then the multigerm is contact equivalent to one of the following normal forms (we indicate only the third component, because the first two components represent the normal form 1 of Lemma 19):

1. $(t \mid 0 ; t)$

2. $(t \mid 0, t ; 0,0)$

3. $\left(t^{m} \mid 0 ; t\right)(m>1)$;

4. $\left(t^{m} \mid 0, t ; 0,0\right)(m>1)$.

Proof. 1) First, we assume that the third component is also transversal to the contact hyperplane. We project the second and the third component to the subspace $\{z=0\}$ along the $z$-axis. A symplectic transformation and, possibly, right changes bring the projection either to the form $\left(\left(t_{1} ; 0\right),\left(0 ; t_{2}\right)\right)$, or to the form $\left(\left(t_{1}, 0 ; 0,0\right),\left(0, t_{2} ; 0,0\right)\right)$. We lift the symplectomorphism to a contact diffeomorphism.

We start with the first possibility. Then we can bring the multigerm to the form

$$
\left((t \mid 0 ; 0),\left(t_{1}+o\left(t_{1}\right) \mid t_{1} ; 0\right),\left(t_{2}+o\left(t_{2}\right) \mid 0 ; t_{2}\right)\right) .
$$

Right changes bring the multigerm to the form

$$
\left((t \mid 0 ; 0),\left(t_{1} \mid t_{1}+o\left(t_{1}\right) ; 0\right),\left(t_{2} \mid 0 ; t_{2}+o\left(t_{2}\right)\right)\right) .
$$

Now we show how to eliminate the perturbations $o\left(t_{i}\right)$. Assume that we have already eliminated all terms of order less than $m(m>1)$. We consider the $m$-jet:

$$
\left((t \mid 0 ; 0),\left(t_{1} \mid t_{1}+a t_{1}^{m} ; 0\right),\left(t_{2} \mid 0 ; t_{2}+b t_{2}^{m}\right)\right) .
$$


In order to eliminate the monomial $a t_{1}^{m}$, we use the contact Hamiltonian $K=a \cdot p q^{m}$. We easily see that such a change affects neither the first nor the third component. Similarly, in order to eliminate $b t_{2}^{m}$, we take $K=-b \cdot p^{m} q$. This yields the normal form 1 .

Now we consider the second possibility, i.e., the projection can be brought to the form $\left(\left(t_{1}, 0 ; 0,0\right),\left(0, t_{2} ; 0,0\right)\right)$. After that, right changes bring the multigerm to the form

$$
\left((t \mid 0,0 ; 0,0),\left(t_{1} \mid t_{1}+o\left(t_{1}\right), 0 ; 0,0\right),\left(t_{2} \mid 0, t_{2}+o\left(t_{2}\right) ; 0,0\right)\right) .
$$

In order to eliminate the perturbations, we use induction once again. Assume that we have already eliminated all terms of order at most $m-1$. We consider the $m$-jet:

$$
\left((t \mid 0,0 ; 0,0),\left(t_{1} \mid t_{1}+a t_{1}^{m}, 0 ; 0,0\right),\left(t_{2} \mid 0, t_{2}+b t_{2}^{m} ; 0,0\right)\right) .
$$

In order to eliminate the monomial $a t_{1}^{m}$, we use the change generated by the contact Hamiltonian $K=a \cdot p_{1} q_{1}^{m}$. We can do the same for the monomial $b t_{2}^{m}$ with the help of $K=b \cdot p_{2} q_{2}^{m}$. Thus, we have obtained the normal form 2 .

2) Now we assume that the third component touches the contact hyperplane. As before, we project the second and the third component to the $(q ; p)$-space along the $z$ axis. Once again, a symplectic transformation brings the projection to one of the two forms indicated above. Lifting the symplectomorphism to a contact diffeomorphism and performing right changes, we bring the multigerm to one of the following two forms:

$$
\left((t \mid 0 ; 0),\left(t_{1} \mid t_{1}+o\left(t_{1}\right) ; 0\right),\left(t_{2}^{m} \mid 0 ; t_{2}+o\left(t_{2}\right)\right)\right)
$$

or

$$
\left((t \mid 0,0 ; 0,0),\left(t_{1} \mid t_{1}+o\left(t_{1}\right), 0 ; 0,0\right),\left(t_{2}^{m} \mid 0, t_{2}+o\left(t_{2}\right) ; 0,0\right)\right) .
$$

Performing the same contact changes as in the case of the normal forms 1 or 2 (we easily see that the form of the coordinate $z$ is not important for these changes), we obtain the normal forms 3 and 4 .

4.2.2. Now we show that no multigerm $R L$-equivalent to $\left((t, 0),\left(0, t_{1}\right),\left(t_{2}, t_{2}\right)\right)$ is simple from the contact point of view.

Lemma 23. Let $F$ be a multigerm in the contact space. If $F$ is RL-equivalent to $\left((t, 0),\left(0, t_{1}\right),\left(t_{2}, t_{2}\right)\right)$, then $F$ is not simple.

Proof. We easily see that if all three components are transversal to the contact hyperplane at the origin (which is true in the generic case), then we can bring the 1-jet to the form

$$
\left((t \mid 0 ; 0),\left(t_{1} \mid t_{1} ; 0\right),\left(\alpha t_{2} \mid t_{2} ; 0\right)\right) \text {. }
$$

After this, direct computations show that the parameter $\alpha$ is a modulus.

4.2.3. Finally, we prove that if a multigerm has at least 4 components, then it is not simple from the contact point of view. It is clear that in the generic case the multigerm must be $R L$-equivalent to

$$
((t, 0,0,0),(0, t, 0,0),(0,0, t, 0),(0,0,0, t)),
$$

and all of its components are transversal to the contact hyperplane at the origin. Then we easily see that in the generic case such a multigerm can be brought to the form

$$
\left((t \mid 0,0 ; 0,0),\left(t_{1} \mid t_{1}, 0 ; 0,0\right),\left(t_{2} \mid 0,0 ; t_{2}, 0\right),\left(t_{3} \mid \alpha t_{3}, t_{3} ; \beta t_{3}, 0\right)\right) .
$$

We prove that, indeed, the parameters $\alpha$ and $\beta$ are moduli. It is clear that we can use only the changes obtained via multiplying the coordinates by coefficients. We consider the behavior of the coordinates $z, q_{1}$, and $p_{1}$. (The other coordinates are of no interest to us.) We must control the behavior of $1+2+2+3=8$ parameters, while the dimension of the corresponding group is at most 4 (right changes) +2 (left changes) $=6$. We have thus proved the following assertion. 
Lemma 24. If a multigerm has at least 4 components, then it is not simple from the contact point of view.

\section{§5. Singularities of multigerms COntaining Singular components}

We assume that a simple multigerm contains a singular component. Then Lemmas 21 and 23 imply that the multigerm contains two components, and one of them (say, the first) is regular and, furthermore, transversal to the contact hyperplane. Then we can assume that this component already has the standard form $(t \mid 0, \ldots, 0 ; 0, \ldots, 0)$.

5.1. First, we suppose that the singular component has multiplicity 2 , and that the singular and nonsingular components do not touch each other. Up to $R L$-equivalence, there are two such multigerms: $\left((t, 0,0),\left(0, t^{2}, t^{2 m+1}\right)\right)$ and $\left((t, 0),\left(t^{2 m+1}, t^{2}\right)\right)$.

Lemma 25. Let $F$ be a multigerm in the contact space. Assume that $F$ is $R L$-equivalent to $\left((t, 0,0),\left(0, t^{2}, t^{2 m+1}\right)\right)$, and that both components are transversal to the contact hyperplane. Then $F$ is equivalent to one of the following two normal forms:

1. $\left((t \mid 0 ; 0),\left(t^{2} \mid t^{2} ; t^{2 m+1}\right)\right)$;

2. $\left((t \mid 0,0 ; 0,0),\left(t^{2} \mid t^{2}, t^{2 m+1} ; 0,0\right)\right)$.

Proof. It is clear that the 2 -jet can be brought to the form $\left((t \mid 0,0 ; 0,0),\left(t^{2} \mid t^{2}, 0 ; 0,0\right)\right)$.

We consider the $2 k$-jet of our curve $(k>1)$. Obviously, we can bring this jet to the following form (we indicate only the second component):

$$
\left(t^{2} \mid t^{2}+a t^{2 k}, b t^{2 k} ; c t^{2 k}, d t^{2 k}\right) .
$$

A change generated by the contact Hamiltonian $K=p_{i} q_{1}^{k}$ eliminates the perturbation in the coordinate $q_{i}$. We eliminate the monomial $d t^{2 k}$ with the help of $K=-d \cdot q_{1}^{k} q_{2}$. Finally, we eliminate the monomial $c t^{2 k}$ with the help of $K=-c \cdot q_{1}^{k+1}$. Observe that none of these changes affects the first (regular) component.

We consider the $(2 m+1)$-jet. Clearly, we can bring it either to the form

$$
\left(t^{2} \mid t^{2}, 0 ; t^{2 m+1}, 0\right)
$$

or to the form

$$
\left(t^{2} \mid t^{2}, t^{2 m+1} ; 0,0\right) \text {. }
$$

1) We start with the first possibility. Clearly, in this case, the multigerm lies in a three-dimensional submanifold, and the induced structure is also contact. So, we bring our curve to a normal form in this space and then apply the contact version of the Darboux-Givental theorem. The corresponding right change brings the $(2 m+2 k+1)$-jet to the form

$$
\left(t^{2} \mid t^{2}+a t^{2 m+2 k+1} ; t^{2 m+1}+b t^{2 m+2 k+1}\right) .
$$

Using the contact Hamiltonian $K=a \cdot p_{1}^{2} q_{1}^{k} / 2$, we eliminate the monomial $a t^{2 m+2 k+1}$. In order to compensate for the perturbation in the coordinate $p_{1}$, we use two contact Hamiltonians $K_{1}=p_{1} q_{1}^{k+1}$ and $K_{2}=p_{1} q_{1}^{k} z$. The action of the corresponding vector fields on our $(2 m+2 k+1)$-jet yields the vectors

$$
\left(0 \mid t^{2 k+2} ;-(k+1) t^{2 m+2 k+1}\right) \text { and }\left(0 \mid t^{2 k+2} ;-k t^{2 m+2 k+1}\right),
$$

respectively, and the vector $\left(0 \mid 0 ; b t^{2 m+2 k+1}\right)$ is their linear combination. Thus, we have obtained the normal form 1 .

2) Now we assume that the $(2 m+1)$-jet can be brought to the form

$$
\left(t^{2} \mid t^{2}, t^{2 m+1} ; 0,0\right) \text {. }
$$


Then we can assume that our multigerm lies in a five-dimensional contact space with coordinates $\left(z \mid q_{1}, q_{2} ; p_{1}, p_{2}\right)$. A right change brings the $(2 m+2 k+1)$-jet of the multigerm to the form

$$
\left(t^{2} \mid t^{2}+a_{1} t^{2 m+2 k+1}, t^{2 m+1}+a_{1} t^{2 m+2 k+1} ; b t^{2 m+2 k+1}, c t^{2 m+2 k+1}\right) .
$$

We eliminate the perturbation in the coordinate $q_{i}$ with the help of the contact Hamiltonian $K=a_{i} \cdot p_{i} q_{1}^{k} q_{2}$. We eliminate the monomial $c t^{2 m+2 k+1}$ with the help of the contact Hamiltonian $K=-c \cdot q_{1}^{k} q_{2}^{2} / 2$. In order to compensate for the perturbation in the coordinate $p_{1}$, we use two contact Hamiltonians $K_{1}=q_{1}^{k+1} q_{2}$ and $K_{2}=q_{1}^{k} q_{2} z$. The action of the corresponding vector fields on our $(2 m+2 k+1)$-jet yields the vectors

$$
\left(0 \mid 0,0 ;-(k+1) t^{2 m+2 k+1},-t^{2 k+2}\right) \text { and }\left(0 \mid 0,0 ;-k t^{2 m+2 k+1},-t^{2 k+2}\right),
$$

respectively, and the vector $\left(0 \mid 0,0 ; b t^{2 m+2 k+1}, 0\right)$ is their linear combination. Thus, we have obtained the normal form 2 .

5.2. Now we assume that our multigerm is $R L$-equivalent to $\left((t, 0),\left(t^{2 m+1}, t^{2}\right)\right)$.

Lemma 26. Let $F$ be a multigerm in the contact space. Assume that $F$ is RL-equivalent to $\left((t, 0),\left(t^{2 m+1}, t^{2}\right)\right)$, and that both components are transversal to the contact hyperplane. Then $F$ is contact equivalent to

$$
\left((t \mid 0 ; 0),\left(t^{2} \mid t^{2}+t^{2 m+1} ; 0\right)\right) .
$$

Proof. Since the multigerm is plane and the tangent space of the germ is transversal to the contact hyperplane, the multigerm is contained in a germ of a two-dimensional submanifold with coordinates $(z, q)$ such that the induced structure is determined by the 1-form $d z$.

Clearly, the 2-jet can be brought to the form $\left(t^{2}, t^{2}\right)$. (Here and until the end of the proof, we indicate only the second component.) A right change brings the curve to the form

$$
\left(t^{2}, t^{2}+\sum_{k=2}^{m} a_{k} t^{2 k}+a_{2 m+1} t^{2 m+1}+o\left(t^{2 m+1}\right)\right) .
$$

First, we successively eliminate the monomials of even degree. This is done with the help of changes of the form $\left(\tilde{z}=z, \tilde{q}=q-a_{k} q^{k}\right)$. Finally, we obtain a $(2 m+1)$-jet, which takes the form $\left(t^{2}, t^{2}+t^{2 m+1}\right)$ after linear changes. The perturbations of even degree are eliminated as indicated above.

We consider the $(2 m+2 k+1)$-jet:

$$
\left(t^{2}, t^{2}+t^{2 m+1}+a t^{2 m+2 k+1}\right) .
$$

We use the change $\left(\tilde{z}=z, \tilde{q}=q-a q^{k}(q-z)\right)$. It is clear that this change does not affect the first component and eliminates the monomial $a t^{2 m+2 k+1}$ in the second component.

5.3. Now we assume that our multigerm is $R L$-equivalent to $\left((t, 0),\left(0, t^{2}, t^{3}\right)\right)$, and that the first component is still transversal to the contact hyperplane, while the second component touches the contact hyperplane.

Lemma 27. Let $F$ be a multigerm in the contact space $\mathbb{C}^{2 n+1}$. Assume that $F$ is $R L$-equivalent to $\left((t, 0),\left(0, t^{2}, t^{3}\right)\right)$. Furthermore, we assume that the first component of $F$ is transversal to the contact hyperplane, the second component touches the contact hyperplane, but the tangent space of the second component does not lie in the contact hyperplane. Then the multigerm is contact equivalent to one of the following two normal 
forms (we indicate only the second component, the first one having the form $(t \mid 0,0 ; 0,0))$ :

1. $\left(t^{3} \mid t^{2} ; t^{3}\right)$;

2. $\left(t^{3} \mid t^{2}, t^{3} ; 0,0\right)$.

Proof. We consider the 3-jet. Under the above assumptions, we can bring this jet to one of the following two forms:

$$
\left(t^{3} \mid t^{2} ; t^{3}\right) \quad \text { or } \quad\left(t^{3} \mid t^{2}, t^{3} ; 0,0\right) .
$$

1) We consider the first possibility. A right change brings the $2 m$-jet $(m>1)$ to the form

$$
\left(t^{3} \mid t^{2}+a t^{2 m-1}+b t^{2 m} ; t^{3}+c t^{2 m}\right) .
$$

We eliminate the monomial $a t^{2 m-1}$ with the help of the contact Hamiltonian $K=$ $a \cdot p^{2} q^{m-2} / 2$. A right change eliminates $b t^{2 m}$. Finally, a change generated by the contact Hamiltonian $K=-c \cdot q^{m+1} /(m+1)$ compensates for the perturbation in the coordinate $p$.

Now we consider the $(2 m+1)$-jet. A right change brings this jet to the form

$$
\left(t^{3} \mid t^{2}+a t^{2 m}+b t^{2 m+1} ; t^{3}+c t^{2 m+1}\right) .
$$

In order to eliminate the monomial $a t^{2 m}$, we take $K=a \cdot p q^{m}$. A right change eliminates $b t^{2 m+1}$. Finally, using the contact Hamiltonian $K=-c \cdot z q^{m} / m$, we compensate for the perturbation in the coordinate $p$. Clearly, this change does not affect the first component. Thus, we have obtained the normal form 1.

2) Now we consider the second possibility: we assume that the 3-jet of the second component has the form $\left(t^{3} \mid t^{2}, t^{3} ; 0,0\right)$. We show that this jet is 3-determined.

Consider the $2 m$-jet $(m>1)$ of such a multigerm. A right change brings this jet to the form

$$
\left(t^{3} \mid t^{2}+a t^{2 m-1}+b t^{2 m}, t^{3}+c t^{2 m} ; d t^{2 m}, e t^{2 m}\right) .
$$

A change generated by the contact Hamiltonian $K=a \cdot p_{1} q_{1}^{m-2} q_{2}$ eliminates the monomial $a t^{2 m-1}$. The perturbation proportional to $t^{2 m}$ in the coordinate $q_{i}$ is eliminated with the help of a contact Hamiltonian proportional to $p_{i} q_{1}^{m}$. In order to compensate for the perturbation in the coordinate $p_{1}$, we take $K=-d \cdot q_{1}^{m+1} /(m+1)$. We can do the same for the coordinate $p_{2}$ with the help of $K=-e \cdot q_{1}^{m} q_{2}$.

Now we consider the $(2 m+1)$-jet:

$$
\left(t^{3} \mid t^{2}+a t^{2 m}+b t^{2 m+1}, t^{3}+c t^{2 m+1} ; d t^{2 m+1}, e t^{2 m+1}\right) .
$$

A change generated by the contact Hamiltonian $K=a \cdot p_{1} q_{1}^{m}$ eliminates the monomial $a t^{2 m}$. The perturbation proportional to $t^{2 m+1}$ in the coordinate $q_{i}$ is eliminated with the help of a contact Hamiltonian proportional to $p_{i} q_{1}^{m-1} q_{2}$. In order to compensate for the perturbation in the coordinate $p_{2}$, we take $K=-e \cdot q_{1}^{m-1} q_{2}^{2} / 2$. We can do the same for the coordinate $p_{1}$ with the help of $K=-d \cdot z q_{1}^{m} / m$. Thus, we have obtained the normal form 2 .

Lemma 28. Let $F$ be a multigerm in the contact space. Assume that $F$ is $R L$-equivalent to $\left((t, 0),\left(t^{3}, t^{2}\right)\right)$, that the first component of $F$ is transversal to the contact hyperplane, and that the second component touches the contact hyperplane. Then the multigerm is contact equivalent to one of the following two normal forms (we indicate only the second component since the first has the same form as in the preceding lemma):

1. $\left(t^{3} \mid t^{2}+t^{3} ; 0\right)$;

2. $\left(t^{3} \mid t^{2} ; 0\right)$. 
Proof. As we did repeatedly before, we find a germ of a two-dimensional submanifold with coordinates $(z, q)$ containing our multigerm. Since the first component is transversal to the contact hyperplane, we can assume that the induced distribution is determined by $d z=0$. It is clear that the 3-jet can be brought to one of the normal forms 1 and 2. Furthermore, after a right change we can assume that the coordinate $z$ of the entire germ (and not only of its 3-jet) is equal to $t^{3}$. We observe that each function of the form $o\left(t^{3}\right)$ can be written as $f(q)+z g(q)$, where $f(q)=O\left(q^{2}\right)$ and $g(0)=0$. Then we use the change

$$
(\tilde{z}=z, \quad \tilde{q}=q-f(q)-z g(q)) .
$$

Obviously, it does not affect the first component and brings the second component to the required form.

Lemma 29. Let $F$ be a multigerm in the contact space. Assume that $F$ is RL-equivalent to $\left((t, 0,0),\left(0, t^{2}, t^{3}\right)\right)$, that the first component of $F$ is transversal to the contact hyperplane, and that the tangent space of the second component is contained entirely in the contact hyperplane. Then the multigerm is not simple from the contact point of view.

Proof. We assume that the first component already has the standard form. In the generic case, the projection of the singular component to the $(p, q)$-space along the $z$-axis has the form $\left(t^{2}+o\left(t^{2}\right) ; t^{3}+o\left(t^{3}\right)\right)$. A symplectic change brings the projection to the form $\left(t^{2} ; t^{3}\right)$. We lift the symplectomorphism corresponding to this change to the contact space. We show that even the 6 -jet of such a multigerm is not simple.

Clearly, in the generic case the 6 -jet of the second component can be brought to the form

$$
\left(t^{4}+a t^{5}+b t^{6} \mid t^{2} ; t^{3}\right) .
$$

First, we eliminate the monomial $a t^{5}$. For this purpose, we apply the homotopy method once again. We must obtain the vector $\left(t^{5}+o\left(t^{5}\right) \mid 0 ; 0\right)$ as a result of the action of elements in the Lie algebra of our group. The tangent space of the $R$-orbit contains the vector $\left(4 t^{5} \mid 2 t^{3} ; 3 t^{4}\right)$. The contact Hamiltonian $K_{1}=q^{3}$ yields the vector $\left(t^{6} \mid 0 ;-3 t^{4}\right)$, and the contact Hamiltonian $K_{2}=p^{2}$ yields the vector $\left(-t^{6} \mid 2 t^{3} ; 0\right)$. We easily see that the required vector is a linear combination of these three vectors. Therefore, the 6 -jet can be brought to the following form (the change can affect the coefficient $b$ ):

$$
\left(t^{4}+b t^{6} \mid t^{2} ; t^{3}\right) \text {. }
$$

Direct computations show that the vector $\left(t^{6} \mid 0 ; 0\right)$ is not contained in the tangent space of the ( $R$ Cont)-orbit.

5.4. Now we assume that the singular component and the nonsingular component touch each other and are transversal to the contact hyperplane. Up to $R L$-equivalence, such a multigerm has the form $\left((t, 0,0),\left(t^{2}, t^{3}, t^{4}\right)\right)$ in the generic case.

Lemma 30. Let $F$ be a multigerm in the contact space. Assume that $F$ is RL-equivalent to $\left((t, 0,0),\left(t^{2}, t^{3}, t^{4}\right)\right)$, and that both components of $F$ are transversal to the contact hyperplane. Then the multigerm is contact equivalent to one of the following two normal forms (we indicate only the second component, the first one having the form $(t \mid 0,0 ; 0,0)$ ):

1. $\left(t^{2} \mid t^{3} ; t^{4}\right)$

2. $\left(t^{2} \mid t^{3}, t^{4} ; 0,0\right)$.

Proof. Clearly, we can bring the 4-jet to one of the two forms indicated above.

1) Suppose that we have obtained a 4 -jet of the form 1 . Then, using the fact that our 4-jet is 4-determined and applying the Darboux-Givental theorem, we can assume that the multigerm lies in the three-dimensional space $\mathbb{C}^{3}$ with coordinates $(z \mid q ; p)$. 
We consider the $(2 m+1)$-jet $(m>1)$. A right change brings this jet to the form

$$
\left(t^{2} \mid t^{3}+a t^{2 m+1} ; t^{4}+b t^{2 m+1}\right) .
$$

A change generated by the contact Hamiltonian $K=a \cdot p q z^{m-1}$ eliminates the monomial $a t^{2 m+1}$, while a change generated by $K=-b \cdot q^{2} z^{m-1} / 2$ compensates for the perturbation in the coordinate $p$.

Now we consider the $2 m$-jet $(m>2)$. A right change brings this jet to the form

$$
\left(t^{2} \mid t^{3}+a t^{2 m} ; t^{4}+b t^{2 m}\right) .
$$

Using the contact Hamiltonian $K=a \cdot p q^{2} z^{m-3}$, we eliminate the perturbation in the coordinate $q$, and a change generated by $K=-b \cdot q^{3} z^{m-3} / 3$ eliminates the perturbation in $p$. Thus, we have obtained the normal form 1.

2) Now we assume that the 4-jet has the form 2. Since this jet is also 4-determined, the Darboux-Givental theorem allows us to assume that the curve lies in the contact space $\mathbb{C}^{5}$ with coordinates $\left(z \mid q_{1}, q_{2} ; p_{1}, p_{2}\right)$.

We consider the $(2 m+1)$-jet of the curve $(m>1)$. Obviously, a right change brings this jet to the form

$$
\left(t^{2} \mid t^{3}+a t^{2 m+1}, t^{4}+b t^{2 m+1} ; c t^{2 m+1}, d t^{2 m+1}\right) .
$$

A change generated by a contact Hamiltonian proportional to $K_{i}=p_{i} q_{1} z^{m-1}$ eliminates the perturbation in the coordinate $q_{i}(i=1,2)$, and a change generated by the contact Hamiltonian $K=-c \cdot q_{1}^{2} z^{m-1} / 2$ eliminates the monomial $c t^{2 m+1}$. In order to eliminate $d t^{2 m+1}$, we take $K=-d \cdot q_{1} q_{2} z^{m-1}$.

Now we consider the $2 m$-jet $(m>2)$. A right change brings this jet to the form

$$
\left(t^{2} \mid t^{3}+a t^{2 m}, t^{4}+b t^{2 m} ; c t^{2 m}, d t^{2 m}\right) .
$$

A change generated by a contact Hamiltonian proportional to $K_{i}=p_{i} q_{1}^{2} z^{m-3} / 3$ eliminates the perturbation in the coordinate $q_{i}(i=1,2)$. Using the contact Hamiltonian $K=-c \cdot q_{1}^{3} z^{m-3} / 3$, we eliminate the perturbation in the coordinate $p_{1}$, and, doing the same for $p_{2}$ with the help of $K=-d \cdot q_{1}^{2} q_{2} z^{m-3}$, we obtain the normal form 2 .

Lemma 31. Let $F$ be a multigerm in the contact space. Assume that $F$ is RL-equivalent to $\left((t, 0),\left(t^{2}, t^{3}\right)\right)$. Then $F$ is contact equivalent to one of the following two normal forms (we indicate only the singular component, the nonsingular one being of the form $(t \mid 0 ; 0)$ ):

1. $\left(t^{2} \mid t^{3}+t^{4} ; 0\right)$;

2. $\left(t^{2} \mid t^{3} ; 0\right)$.

Proof. As we did repeatedly before, we use the fact that our multigerm is plane and the tangent plane of the germ is transversal to the contact hyperplane. On a two-dimensional surface containing our multigerm, we consider the coordinates $(z, q)$ in which the induced structure is determined by the form $d z$. Clearly, we can bring the multigerm to the form

$$
\left((t, 0),\left(t^{2}, t^{3}+a t^{4}+O\left(t^{5}\right)\right) .\right.
$$

If $a \neq 0$, then, multiplying the coordinates and the independent variable by constants, we can make $a=1$. Now we observe that each function of the form $O\left(t^{5}\right)$ can be written as $q f(z, q)$. Performing the change $(\tilde{z}=z, \tilde{q}=q-q f(z, q))$, we obtain the normal form 1 if $a \neq 0$, and the normal form 2 if $a=0$. 
5.5. Now we assume that our multigerm is $R L$-equivalent to $\left((t, 0,0),\left(t^{2}, t^{4}, t^{5}\right)\right)$.

Lemma 32. Let $F$ be a multigerm in the contact space. Assume that $F$ is $R L$-equivalent to $\left((t, 0,0),\left(t^{2}, t^{4}, t^{5}\right)\right)$, and that the tangent vector of the two components is transversal to the contact hyperplane. Then the multigerm is contact equivalent to one of the following three normal forms (we indicate only the singular component, because the nonsingular one has the form $(t \mid 0,0 ; 0,0))$ :

1. $\left(t^{2} \mid t^{4}, 0 ; t^{5}, 0\right)$;

2. $\left(t^{2} \mid t^{4}, t^{5} ; t^{7}, 0\right)$;

3. $\left(t^{2} \mid t^{4}, t^{5} ; 0,0\right)$.

Proof. Obviously, we can bring the 5-jet to one of the following two forms:

$$
\left(t^{2} \mid t^{4}, 0 ; t^{5}, 0\right)
$$

or

$$
\left(t^{2} \mid t^{4}, t^{5} ; 0,0\right) \text {. }
$$

1) We start with the first possibility. We observe that our 5 -jet is 5 -determined (from the viewpoint of $R L$-equivalence). Therefore, applying the Darboux-Givental theorem, we can assume that our multigerm lies in the three-dimensional space $\mathbb{C}^{3}$.

We consider the $2 m$-jet of the multigerm $(m>2)$. A right change brings the jet to the form

$$
\left(t^{2} \mid t^{4}+a t^{2 m} ; t^{5}+b t^{2 m}\right) \text {. }
$$

A change generated by the contact Hamiltonian $a \cdot p q z^{m-2}$ eliminates the monomial $a t^{2 m}$. Using the contact Hamiltonian $K=-b \cdot q_{1}^{2} z^{m-2} / 2$, we eliminate $b t^{2 m}$.

Consideration of the 7 -jet requires a special argument. A right change brings this jet to the form

$$
\left(t^{2} \mid t^{4}+a t^{7} ; t^{5}+b t^{7}\right) .
$$

A change generated by the contact Hamiltonian $K=a \cdot p^{2} z / 2$ eliminates the monomial $a t^{7}$.

Now we pass to the monomial $b t^{7}$. We must verify that the vector $\left(0 \mid 0 ; t^{7}\right)$ lies in the tangent space of the ( $R$ Cont)-orbit. The contact Hamiltonian $K_{1}=p q z$ yields the vector $\left(0 \mid t^{6} ;-t^{7}\right)$, the tangent space of the $R$-orbit contains the vector $\left(2 t^{4} \mid 4 t^{6} ; 5 t^{7}\right)$, and, finally, the contact Hamiltonian $K_{2}=z^{2}$ yields the vector $\left(t^{4} \mid 0 ; 2 t^{7}\right)$. (The action of $K_{2}$ on the nonsingular component yields the vector $\left(t^{2} \mid 0 ; 0\right)$, which is compensated for by a right change.) We easily see that the required vector is a linear combination of the three vectors indicated above.

Now we consider the $(2 m+1)$-jet $(m>3)$. A right change brings this jet to the form

$$
\left(t^{2} \mid t^{4}+a t^{2 m+1} ; t^{5}+b t^{2 m+1}\right) \text {. }
$$

We eliminate the perturbation in the coordinate $q$ with the help of the contact Hamiltonian $K=a \cdot p^{2} z^{m-2} / 2$. In order to eliminate the monomial $b t^{2 m+1}$, we observe that the contact Hamiltonians $K_{1}=p q z^{m-2}$ and $K_{2}=p q^{2} z^{m-4}$ yield the tangent vectors

$$
\left(0 \mid t^{2 m} ;-t^{2 m+1}\right) \text { and }\left(0 \mid t^{2 m} ;-2 t^{2 m+1}\right) .
$$

Obviously, $b t^{2 m+1}$ is eliminated by a contact Hamiltonian proportional to $K_{1}-K_{2}$.

2) Now we consider the second possibility, where the 5 -jet has the form

$$
\left(t^{2} \mid t^{4}, t^{5} ; 0,0\right) \text {. }
$$

As before, applying the Darboux-Givental theorem, we can assume that the multigerm lies in a five-dimensional space. 
We consider the $2 m$-jet $(m>2)$. A right change brings this jet to the following form (we assume that there are no perturbations of smaller degrees):

$$
\left(t^{2} \mid t^{4}+a t^{2 m}, t^{5}+b t^{2 m} ; c t^{2 m}, d t^{2 m}\right) .
$$

A change generated by a contact Hamiltonian proportional to $K_{i}=p_{i} q_{1} z^{m-2}$ eliminates the perturbation in the coordinate $q_{i}$. We eliminate the perturbation in the coordinate $p_{1}$ with the help of the contact Hamiltonian $K=-c \cdot q_{1}^{2} z^{m-2} / 2$. Similarly, we eliminate the perturbation in $p_{2}$ with the help of $K=-d \cdot q_{1} q_{2} z^{m-2}$. Thus, in particular, we have considered the 6-jet.

We pass to the 7-jet. This jet has the form

$$
\left(t^{2} \mid t^{4}+a t^{7}, t^{5}+b t^{7} ; c t^{7}, d t^{7}\right)
$$

We eliminate the perturbation in the coordinate $q_{i}$ with the help of a contact Hamiltonian proportional to $K_{i}=p_{i} q_{2} z$. Similarly, we eliminate the perturbation in $p_{2}$ with the help of $K=-d \cdot q_{2}^{2} z / 2$. Generally speaking, the monomial $c t^{7}$ cannot be "killed". Indeed, the only way to obtain $t^{7}$ in $p_{1}$ is to take $K=q_{1} q_{2} z$. In this way, we obtain the tangent vector $\left(0 \mid 0,0 ;-t^{7},-t^{6}\right)$. However, it is clear that we cannot obtain the monomial $t^{6}$ in the coordinate $p_{2}$ in any other way without changing the nonsingular component. Therefore, if $c \neq 0$, then we obtain the 7 -jet 2 , while if $c=0$, then we obtain the 7 -jet 3 . We easily see that the argument allowing us to eliminate perturbations of degree $2 m$ is valid for both cases.

Now we consider the $(2 m+1)$-jet $(m>3)$. A right change brings this jet to the following form $(\varepsilon=0$ or 1$)$ :

$$
\left(t^{2} \mid t^{4}+a t^{2 m+1}, t^{5}+b t^{2 m+1} ; \varepsilon t^{7}+c t^{2 m+1}, d t^{2 m+1}\right) .
$$

A change generated by a contact Hamiltonian proportional to $K_{i}=p_{i} q_{2} z^{m-2}$ eliminates the perturbation in the coordinate $q_{i}$. Using the contact Hamiltonian $K=-d \cdot q_{2}^{2} z^{m-2} / 2$, we eliminate the perturbation in the coordinate $p_{2}$. To compensate for the perturbation in $p_{1}$, we use two contact Hamiltonians $K_{1}=-q_{1} q_{2} z^{m-2}$ and $K_{2}=-q_{1}^{2} q_{2} z^{m-4}$. Their action on our one-parameter family yields the vectors

$$
\left(0 \mid 0,0 ; t^{2 m+1}, t^{2 m}\right) \text { and }\left(0 \mid 0,0 ; 2 t^{2 m+1}, t^{2 m}\right) \text {, }
$$

respectively. Obviously, the required vector $\left(0 \mid 0,0 ; t^{2 m+1}, 0\right)$ is their linear combination. The corresponding linear combination of $K_{1}$ and $K_{2}$ yields the required contact Hamiltonian.

5.6. Now we show that our list of simple multigerms is complete.

Lemma 33. If a multigerm $F$ is $R L$-equivalent to $\left((t, 0,0),\left(t^{2}, t^{4}+t^{5}, t^{7}\right)\right)$, then $F$ is not simple from the contact point of view.

Proof. In the generic case, the tangent vector of both components of the multigerm is transversal to the contact hyperplane. Therefore, we can assume that the nonsingular component has the form $(t \mid 0, \ldots, 0 ; 0, \ldots, 0)$. Then the 5 -jet of the singular component can be brought to the form $\left(t^{2} \mid t^{4}+t^{5}, 0, \ldots, 0 ; 0,0, \ldots, 0\right)$. The proof of the preceding lemma shows that the 6 -jet of the multigerm remains the same.

Now we consider the 7-jet. Clearly, in the generic case the jet can be brought to the form

$$
\left(t^{2} \mid t^{4}+a t^{5}, b t^{7} ; t^{7}, 0\right) .
$$

(We do not indicate the regular component, which has the standard form.) We set $S(p)=p_{1} p_{2}$ and use the change (3.4), which brings the second component to the form

$$
\left(t^{2} \mid t^{4}+a t^{5} ; t^{7}\right) .
$$


This 7-jet is not simple any more, the parameter $a$ being a modulus. Indeed, it is clear that we can use only the changes obtained via multiplying the coordinates and the independent variable by constants. However, the dimension of the group of such changes is equal to 3 , while we must control the behavior of 4 parameters (the coefficients).

5.7. Finally, we show that if a multigerm $F$ contains a nonsingular component and a component of multiplicity 3 , then $F$ is not simple from the contact point of view. Up to $R L$-equivalence, $F$ has the form $\left((t, 0,0,0),\left(0, t^{3}, t^{4}, t^{5}\right)\right)$ in the generic case. It is also clear that in the generic case both components of $F$ are transversal to the contact hyperplane. Then we can bring the 3 -jet of $F$ to the form

$$
\left((t \mid 0 ; 0),\left(t^{3} \mid t^{3} ; 0\right)\right) \text {. }
$$

In the generic case, we can bring the 4 -jet of $F$ to the form

$$
\left((t \mid 0,0 ; 0,0),\left(t^{3} \mid t^{3}, a t^{4} ; t^{4}, 0\right)\right) .
$$

We use the change (3.4) with $S=a \cdot p_{1} p_{2}$, which brings the 4-jet of $F$ to the form

$$
\left((t \mid 0,0 ; 0,0),\left(t^{3} \mid t^{3}, 0 ; t^{4}, 0\right)\right) .
$$

Now we consider the 5 -jet. In the generic case, we can bring it to the form

$$
\left((t \mid 0,0 ; 0,0),\left(t^{3} \mid t^{3}+a t^{5}, t^{5} ; t^{4}+b t^{5}, 0\right)\right) .
$$

The monomial $t^{5}$ in the coordinate $q_{2}$ does not allow us to eliminate similar monomials in the coordinates $q_{1}$ and $p_{1}$. Indeed, the action of the contact Hamiltonian $K_{1}=-q_{1} q_{2}$ on our 5 -jet yields the tangent vector $\left(0 \mid 0,0 ; t^{5}, t^{3}+a t^{5}\right)$, and the monomial $t^{3}$ cannot be compensated for by anything. Similarly, the contact Hamiltonian $K_{2}=p_{1} q_{2}$ yields the tangent vector $\left(0 \mid t^{5}, 0 ; 0,-t^{4}-b t^{5}\right)$, and the monomial $-t^{4}$ cannot be compensated for by anything. We easily see that right changes cannot be used also (because they generate monomials of degrees 4 or 5 in the coordinate $z$ ). The remaining changes are obtained via multiplying the coordinates $z, q_{1}$, and $p_{1}$ and the independent variable by constants. However, the dimension of the group of such changes is equal to 3 , while we must control the behavior of 5 parameters. Thus, we have proved the following assertion.

Lemma 34. No multigerm containing a nonsingular component and a component of multiplicity 3 is simple from the contact point of view.

\section{REFERENCES}

[1] J. W. Bruce and T. J. Gaffney, Simple singularities of mappings $(\mathbb{C}, 0) \rightarrow\left(\mathbb{C}^{2}, 0\right)$, J. London Math. Soc. (2) 26 (1982), 465-474. MR684560 (84d:32012)

[2] C. G. Gibson and C. A. Hobbs, Simple singularities of space curves, Math. Proc. Cambridge Philos. Soc. 113 (1993), no. 2, 297-310. MR1198413 (94a:58026)

[3] V. I. Arnol'd, Simple singularities of curves, Trudy Mat. Inst. Steklov. 226 (1999), 27-35; English transl., Proc. Steklov Inst. Math. 1999, no. 3 (226), 20-28. MR.1782550 (2001j:32025)

[4] P. A. Kolgushkin and R. R. Sadykov, Classification of simple multigerms of curves, Uspekhi Mat. Nauk 56 (2001), no. 6, 153-154; English transl., Russian Math. Surveys 56 (2001), no. 6, 1166-1167. MR 1886730

[5] - Simple singularities of multigerms of curves, Rev. Mat. Complut. 14 (2001), no. 2, 311-344; math.AG/0012040. MR:1871300(2002h:14002)

[6] V. I. Arnold, First steps of local symplectic algebra, Differential Topology, Infinite-Dimensional Lie Algebras, and Applications, Amer. Math. Soc. Transl. Ser. 2, vol. 194, Amer. Math. Soc., Providence, RI, 1999, pp. 1-8. MR1729356 (2001h:58055)

[7] P. A. Kolgushkin, Classification of simple multigerms of curves in a space with symplectic structure, Algebra i Analiz 15 (2003), no. 1, 148-183; English transl., St. Petersburg Math. J. 15 (2004), no. 1, 103-126. MR.1979720 (2004g:58054)

[8] V. I. Arnold, First steps of local contact algebra, Canad. J. Math. 51 (1999), no. 6, 1123-1134. MR1756874 (2001c:58039) 
[9] _ Mathematical methods of classical mechanics, "Nauka", Moscow, 1974; English transl., Grad. Texts in Math., vol. 60, Springer-Verlag, New York, 1989. MR0474390 (57:14032); MR0997295 (90c:58046)

[10] V. I. Arnol'd and A. B. Givental', Symplectic geometry, Itogi Nauki i Tekhniki Sovrem. Probl. Mat. Fundam. Naprav., vol. 4, VINITI, Moscow, 1985, pp. 5-139; English transl., Encyclopaedia Math. Sci., vol. 4, Springer-Verlag, Berlin-New York, 1990, pp. 1-136. MR842908 (88b:58044) MR.1042758(90j:58039)

Mechanics and Mathematics Department, Moscow State University, Moscow 119899, Russia

E-mail address: kolgush@mccme.ru

Received 10/MAR/2005

Translated by N. YU. NETSVETAEV 